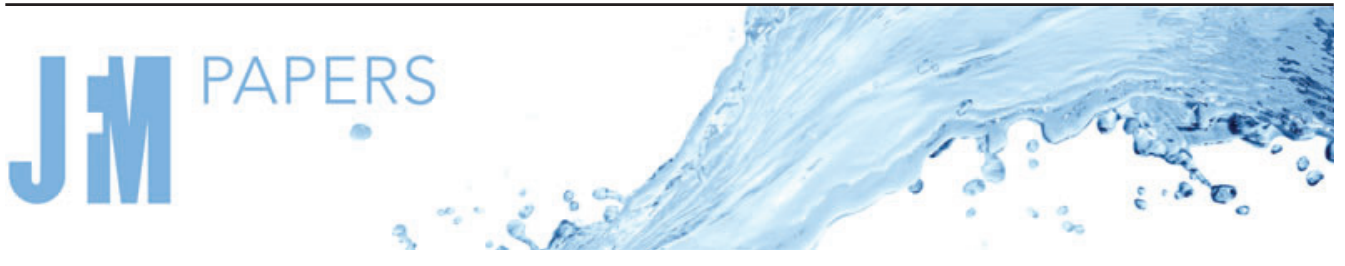

\title{
About the influences of compressibility, heat transfer and pressure gradients in compressible turbulent boundary layers
}

\author{
Christoph Wenzel ${ }^{1,} \dagger$, Tobias Gibis ${ }^{1}$ and Markus Kloker ${ }^{1}$ \\ ${ }^{1}$ Institute of Aerodynamics and Gas Dynamics, University of Stuttgart, 70569 Stuttgart, Germany \\ (Received 31 March 2021; revised 21 July 2021; accepted 6 October 2021)
}

This paper presents a comprehensive analysis of the momentum and energy transfer in compressible turbulent boundary layers based on integral identities. By considering data obtained from direct numerical simulations for a wide parameter range, the superordinate influences of compressibility, wall heat transfer and pressure gradient on the terms of the governing equations are identified and visualized. This allows us both to determine to what degree cases corresponding to different Mach number, heat transfer and pressure-gradient conditions have physically comparable behaviour and to design turbulent boundary-layer cases with specific sought-after behaviour. To this end, newly formulated identities for the skin-friction coefficient $c_{f}$ and the specific heat-transfer coefficient $c_{h}$ from wall-normal integrals based on the non-dimensional compressible momentum and total-enthalpy equations are derived and evaluated. As the individual terms of the resulting identities stay formally close to the terms of the governing equations, the integral analysis further allows the evaluation of common arguments derived from the 'strong' Reynolds analogy from an integral perspective. A particular formulation of the Eckert number $E c$ is proposed as a similarity parameter, mapping cases with different Mach numbers and wall heat transfer conditions.

Key words: compressible boundary layers, compressible turbulence, turbulent boundary layers

\section{Introduction}

In compressible turbulent boundary layers (TBLs), the interaction of compressibility, wall heat transfer and pressure gradient results in a complex parameter space. Thus an intuitive statement about how the single terms of the governing equations behave for different conditions is difficult to formulate. As only one example, it is well known that some

$\dagger$ Email address for correspondence: wenzel@iag.uni-stuttgart.de 


\section{Wenzel, T. Gibis and M. Kloker}

aspects of our simplified understanding of the analogy between momentum and energy transfer in TBLs have to be modified for 'strongly' cooled cases; see e.g. Zhang, Duan \& Choudhari (2018). However, if cases at various Mach numbers are considered, it is not at all obvious what 'strongly' actually means for each Mach-number case. Hence, as all the influences of compressibility, wall heat transfer and pressure gradient lead to a shift in the relevance of the single terms in the overall transfer processes with respect to each other, the question of comparability between certain cases always arises.

For describing the complex interrelation between the momentum and energy transfer within the TBL, the 'strong' Reynolds analogy (SRA) provides an essential building block, giving us a reasonably good understanding of how the compressible TBL behaves. Nearly all theoretical descriptions of compressible TBLs are related in some way to the idea of the SRA - for example, Morkovin's hypothesis that compressible zero-pressure-gradient (ZPG) TBLs behave similarly to 'strangely' heated incompressible ZPG TBLs for large regions of the boundary layer (Smits \& Dussauge 2006). Essentially, the main idea of the SRA is simple. If the Prandtl number $\operatorname{Pr}=1$, the (dimensionless) $x$-momentum and total-enthalpy boundary-layer equations can be rewritten in a similar form,

$$
\begin{gathered}
\bar{\rho} \tilde{u} \frac{\partial \tilde{u}}{\partial x}+\bar{\rho} \tilde{v} \frac{\partial \tilde{u}}{\partial y}+\frac{\gamma}{M^{2}} \frac{\partial \bar{p}}{\partial x}=\frac{\partial}{\partial y}\left[\frac{1}{R e} \tilde{\mu} \frac{\partial \tilde{u}}{\partial y}-\bar{\rho} \widetilde{u}^{\prime \prime} v^{\prime \prime}\right], \\
\bar{\rho} \tilde{u} \frac{\partial \tilde{h}_{0}}{\partial x}+\bar{\rho} \tilde{v} \frac{\partial \tilde{h}_{0}}{\partial y}=\frac{\partial}{\partial y}\left[\frac{1}{R e} \tilde{\mu} \frac{\partial \tilde{h}_{0}}{\partial y}-\bar{\rho} \widetilde{h}_{0}^{\prime \prime} v^{\prime \prime}\right] .
\end{gathered}
$$

Hereinafter, for an arbitrary variable $f$ Reynolds (ensemble) averages are denoted by an overbar, $\bar{f}$, density-weighted Favre averages are denoted by a tilde, $\tilde{f}=\overline{\rho f} / \bar{\rho}$, and values that do not have a fluctuation component are denoted without an overbar/tilde; fluctuations around the Reynolds and Favre averages are denoted by single and double primes, $f^{\prime}=f-\bar{f}$ and $f^{\prime \prime}=f-\tilde{f}$, respectively. The similarity between the momentum and total-enthalpy equations leads to the assumption that individual terms of the momentum and total-enthalpy equations can be directly coupled with each other under a 'strong' assumption of direct proportionality of total-enthalpy and velocity fluctuations, $h_{0}^{\prime \prime}=$ $E c k_{1} u^{\prime \prime}$, where $k_{1}=-1 /(\operatorname{Pr} E c)\left(\bar{q}_{w} / \bar{\tau}_{w}\right)$ is a proportionality constant; see Morkovin (1961), Gaviglio (1987) and Zhang et al. (2014). Here, $\bar{\tau}_{w}$ is the mean wall shear stress and $\bar{q}_{w}$ the wall heat flux in the wall-normal direction $y$. The assumed proportionality between $h_{0}^{\prime \prime}$ and $u^{\prime \prime}$ can be interpreted as a turbulence model for the energy equation, which allows calculation of the mean-temperature profile based on the mean-velocity profile as a linear relation of both profiles; see e.g. Walz (1959). An obvious weakness of the SRA becomes apparent when considering adiabatic walls $\left(\bar{q}_{y, w}=0\right)$, for which $k_{1}$ and thus $h_{0}^{\prime \prime}$ becomes zero. This implication has been proven incorrect since the total-temperature fluctuations $h_{0}^{\prime \prime}$ are of the same order of magnitude as the temperature fluctuations; see e.g. Guarini et al. (2000). Nevertheless, the errors made in some predictions by the SRA are often surprisingly small but can also become large for selected parameter ranges. In recent years, several improvements have been proposed, which have proven to be very powerful for various flow conditions; see e.g. Duan \& Martin (2011) and Zhang et al. (2014).

For the isolated influence of the (non-hypersonic) Mach number under adiabatic conditions, our present knowledge can describe the most fundamental behaviour reasonably well. According to Morkovin (1961), the effects of compressibility are essentially passive, allowing a large number of compressible fluid-flow quantities to be transformed to incompressible reference cases. As a consequence, Mach-number influences on the SRA are only minor, and the success of the Walz relation in determining 


\section{Momentum and energy transfer in compressible TBLs}

the temperature profile from a given velocity profile is almost Mach-number independent. For compressible TBLs, the most complete studies that work out the effect of the Mach number under adiabatic wall conditions can be found in Guarini et al. (2000), Duan, Beekman \& Martin (2011), Bernardini \& Pirozzoli (2011) and Wenzel et al. (2018), to mention a few; all those are based on direct numerical simulation (DNS) data.

From a theoretical perspective, the influence of wall heat transfer can imitate a temperature profile that (for large regions of the boundary layer) is comparable to that caused by a change of the Mach number, and vice versa. In contrast to Mach-number influences, however, the effect of wall heat transfer can affect the TBL behaviour to a much greater extent (strong heating/cooling), resulting in conditions that are difficult to predict. Since the temperature gradient changes its sign along the wall-normal direction inside the TBL for cooled cases (negative at the wall, positive further outside), the signs of the conductive and turbulent heat transfer also change within the boundary layer. Consequently, certain characteristics such as the correlation between temperature and velocity fluctuations can behave differently from adiabatic cases, see Zhang et al. (2014), thus challenging the basic assumption made in the SRA of a direct proportionality between velocity and total-enthalpy fluctuations.

In contrast to the effects of Mach number and wall heat transfer, our knowledge about the influence of streamwise pressure gradients on the SRA's validity is scarce. This does not mean that we expect our general understanding of the SRA to not apply in these cases; see Gaviglio (1987). However, since the understanding has to be based on evidence, one cannot simply estimate to what extent it still applies to pressure-gradient cases without specific evidence/validation. For general cases with the combined influence of (strong) pressure gradients and heat transfer, Fernholz \& Finley (1980) and Gaviglio (1987) have pointed out that it appears difficult to incorporate both influencing factors adequately. For the special class of self-similar cases with 'moderate' pressure-gradient strength, it was shown by Wenzel et al. (2019) that the basic assumptions of Morkovin (and hence implicitly the SRA) still work in good approximation. This behaviour is supported by the self-similarity analysis in Gibis et al. (2019), which suggests that many conclusions found for adiabatic self-similar cases translate to non-adiabatic cases if the heat-transfer distribution is appropriately designed. These findings are further evidenced in Wenzel et al. (2021), where the Reynolds analogy factor $s=2 c_{h} / c_{f}$ was shown to be only weakly influenced by the local Mach number for the weakly non-adiabatic self-similar cases considered. Here, $c_{f} \equiv \bar{\tau}_{w} /\left(\rho_{e} u_{e}^{2} / 2\right)$ is the skin-friction coefficient and $c_{h} \equiv \bar{q}_{w} /\left(\rho_{e} u_{e} c_{p}\left(T_{w}-\bar{T}_{r}\right)\right)$ the specific heat-transfer coefficient, i.e. the Stanton number. As the Reynolds analogy factor can be interpreted as an integral measure of the SRA, see $\S 3$, the only weak Mach-number dependence of $s$ was interpreted as an indication that the main principles of the SRA will work comparably to the ZPG cases, at least for the near-adiabatic self-similar pressure-gradient cases considered.

\subsection{Methodology and objectives of this study}

From the current state of the literature, it can be concluded that the isolated influences of Mach number, heat transfer and, in some part, the pressure gradient on the momentum and energy transfer in TBLs are described to a reasonably good extent. If more than one parameter is changed simultaneously, however, it is not apparent how the governing equations (and hence the SRA's validity) are affected. This study's primary goal is to provide a broad view on the behaviour of compressible TBLs in the complex parameter space, visualizing the effects of several influencing parameters on the single terms of the 


\section{Wenzel, T. Gibis and M. Kloker}

governing equations in a more tangible fashion. This view enables both an evaluation of the comparability of different TBLs under different conditions and the design of TBL cases with specific sought-after behaviour. For this purpose, direct numerical simulations of both sub- and supersonic flat-plate TBLs are conducted at various wall-temperature conditions in both zero and adverse pressure-gradient conditions (self-similar cases). The obtained data are analysed using an integral method, where the momentum and total-enthalpy equations are integrated in the wall-normal direction. Consequently, the dependency of the individual terms on the wall-normal coordinate is eliminated, which in turn allows a scalar and thus compact perspective over the streamwise direction. This approach results in integral identities for $c_{f}$ and $c_{h}$, which hence quantify the integral effects of the overall transfer processes occurring inside the boundary layer. Both identities rely on the same mathematical approach as the Fukagata, Iwamoto \& Kasagi (2002), or FIK, identity, which provides a tool for analysing the $c_{f}$ distribution under both compressible and incompressible conditions. To the authors' best knowledge, the identity for $c_{h}$ is the first of this kind for spatially developing boundary layers. Furthermore, by utilizing the Reynolds analogy factor $s=2 c_{h} / c_{f}$, the two identities are coupled with each other, allowing insight into the analogy between momentum and energy transfer, and hence some aspects of the SRA.

The study is structured as follows: first, the DNS data are summarized in $\S 2$. To allow for a clear understanding of the integral analysis used, its main idea and its derivation are introduced in $\S 3$. To provide an understanding of the raw data used, the spatial evolutions of the integral identities of $c_{f}$ and $c_{h}$ are described qualitatively in $\S 4$. With the goal of examining the isolated effects of compressibility, heat transfer and pressure gradients on the respective identities, the $c_{f}$ and $c_{h}$ identities are analysed in more detail in $\S \S 5$ and 6 , respectively. Further remarks about resulting consequences and implications are given in $\S 7$, and the conclusions are summarized in $\S 8$.

\section{Simulation details}

This study is based on DNS results from self-similar compressible TBLs with adverse pressure gradients (APGs) and ZPGs for inflow Mach numbers of $M_{\infty, 0}=0.3,0.5,0.95$ and 2.0. All cases are computed on the DNS set-ups presented in Wenzel et al. (2019), one each for sub- and supersonic cases. To allow for a meaningful determination of $c_{h} \equiv$ $\bar{q}_{y, w} /\left(\rho_{e} u_{e} c_{p}\left(T_{w}-\bar{T}_{r}\right)\right)$, all cases have been recomputed with near-adiabatic fixed wall temperatures $T_{w}$; some of the cases have been discussed already in Wenzel et al. (2021). To enable reliable conclusions to be drawn about the influence of the Eckert number $E c=$ $(\gamma-1) M_{e}^{2} T_{e} /\left(\bar{T}_{r}-T_{w}\right)$ on the investigations, the wall temperature $T_{w}$ was altered to $2 \mathrm{~K}$, $10 \mathrm{~K}$ and $20 \mathrm{~K}$ above and $10 \mathrm{~K}$ below the adiabatic wall temperature $\bar{T}_{r}$ taken from the fully adiabatic reference cases in Wenzel et al. (2019). For the $M_{\infty, 0}=0.3$ and $M_{\infty, 0}=$ 0.95 cases, new adiabatic reference cases were computed. Note that the adiabatic wall temperature is a function of $x$ for cases with pressure gradient, and so is the prescribed wall temperature. Besides, a strongly cooled ZPG case at $M_{\infty}=2.0$ and $\left(T_{w}-\bar{T}_{r}\right)=-308 \mathrm{~K}$ has been simulated, which was specially designed to verify observed trends; see $\S 6$.

The kinematic Rotta-Clauser parameter $\beta_{K}=\left(\delta_{K}^{*} / \bar{\tau}_{w}\right)\left(\mathrm{d} p_{e} / \mathrm{d} x\right)$, constructed with the incompressible form of the displacement thickness $\delta_{K}^{*}=\int_{0}^{\delta 99}\left(1-\bar{u} / u_{e}\right) \mathrm{d} y$ as a length scale, allows a comparison of pressure-gradient effects between compressible and incompressible cases; see Wenzel et al. (2019) and Gibis et al. (2019). Boundary-layer edge values (index $e$ ) are determined at the wall-normal position where the spanwise vorticity $\bar{\omega}_{z}$ drops below $10^{-6}$ of its wall value, similar to Spalart \& Strelets (2000). Relevant 


\begin{tabular}{|c|c|c|c|c|c|c|c|}
\hline & Case & $M_{e}$ & $E c$ & $T_{w} / T_{e}$ & $T_{w} / \bar{T}_{r}$ & $R e_{\tau}$ & $\operatorname{Re}_{\theta}$ \\
\hline (1) & $\mathrm{iZPG}_{M=0.3}^{+10 \mathrm{~K}}$ & 0.30 & -1.03 & 1.051 & 1.034 & $274 / 726$ & $691 / 2342$ \\
\hline (2) & $\mathrm{iZPG}_{M=0.5}^{+2 \mathrm{~K}}$ & 0.50 & -13.33 & 1.052 & 1.007 & $271 / 731$ & $695 / 2303$ \\
\hline (3) & $\mathrm{iZPG}_{M=0.5}^{+10 \mathrm{~K}}$ & 0.50 & -2.84 & 1.079 & 1.034 & $263 / 714$ & $688 / 2298$ \\
\hline (4) & $\mathrm{cZPG}_{M=0.95}^{+10 \mathrm{~K}}$ & 0.95 & -9.35 & 1.196 & 1.033 & $228 / 612$ & $665 / 2200$ \\
\hline (5) & $\mathrm{cZPG}_{M=2.0}^{-308 \mathrm{~K}}$ & 2.00 & 1.50 & 0.642 & 0.376 & $682 / 2018$ & $1004 / 3469$ \\
\hline (6) & $\mathrm{cZPG}_{M=2.0}^{+20 \mathrm{~K}}$ & 2.00 & -23.10 & 1.781 & 1.041 & $146 / 478$ & $618 / 2509$ \\
\hline (7) & $\mathrm{iAPG}_{\beta_{K}=0.2}^{+10 \mathrm{~K}}$ & $0.46 / 0.43$ & $-2.39 /-2.12$ & $1.071 / 1.066$ & 1.033 & $432 / 726$ & $1406 / 2587$ \\
\hline (8) & $\mathrm{iAPG}_{\beta_{K}=0.6}^{-10 \mathrm{~K}}$ & $0.41 / 0.36$ & $2.00 / 1.52$ & $0.994 / 0.988$ & 0.966 & $480 / 812$ & $1670 / 3071$ \\
\hline (9) & $\mathrm{iAPG}_{\beta_{K}=0.6}^{+2 \mathrm{~K}}$ & $0.39 / 0.36$ & $-8.99 /-8.18$ & $1.032 / 1.028$ & 1.006 & $561 / 783$ & $2107 / 3058$ \\
\hline (10) & $\mathrm{iAPG}_{\beta_{K}=0.6}^{+10 \mathrm{~K}}$ & $0.41 / 0.36$ & $-1.97 /-1.56$ & $1.062 / 1.055$ & 1.032 & $443 / 759$ & $1660 / 3071$ \\
\hline (11) & $\mathrm{iAPG}_{\beta_{K}=1.0}^{+10 \mathrm{~K}}$ & $0.39 / 0.33$ & $-1.76 /-1.30$ & $1.058 / 1.051$ & 1.032 & $449 / 757$ & $1856 / 3463$ \\
\hline (12) & $\mathrm{cAPG}_{\beta_{\mathrm{K}}=0.6}^{+20 \mathrm{~K}}$ & $1.69 / 1.40$ & $-18.82 /-14.59$ & $1.561 / 1.395$ & 1.040 & $394 / 839$ & $1988 / 4270$ \\
\hline
\end{tabular}

Table 1. Summary of boundary-layer parameters for the DNS cases. All data are evaluated at the beginning and the end of the self-similar regions. Given parameters are the boundary-layer edge Mach number $M_{e}$, the Eckert number $E c$, the temperature ratios $T_{w} / T_{e}$ and $T_{w} / \bar{T}_{r}$ and the Reynolds numbers $R e_{\tau}=\bar{\rho}_{w} u_{\tau} \delta_{99} / \bar{\mu}_{w}$ and $\operatorname{Re}_{\theta}=\rho_{e} u_{e} \theta / \mu_{e}$. As $T_{w} / \bar{T}_{r}$ only slightly varies for the APG cases through the domain, an average value is given.

boundary-layer properties, evaluated at the beginning and the end of the self-similar regions used, are summarized in table 1 . Given parameters are the Mach number at the boundary-layer edge $M_{e}$, the Eckert number $E c$, the Reynolds numbers $R e_{\tau}=\bar{\rho}_{w} u_{\tau} \delta_{99} / \bar{\mu}_{w}$ and $\operatorname{Re}_{\theta}=\rho_{e} u_{e} \theta / \mu_{e}$, the wall-to-edge temperature ratio $T_{w} / T_{e}$ and the wall-to-adiabatic temperature ratio $T_{w} / \bar{T}_{r}$. The displacement and momentum thicknesses used are $\delta^{*}=$ $\int_{0}^{\delta_{99}}\left(1-(\bar{\rho} \bar{u}) /\left(\rho_{e} u_{e}\right)\right) \mathrm{d} y$ and $\theta=\int_{0}^{\delta_{99}}(\bar{\rho} \bar{u}) /\left(\rho_{e} u_{e}\right)\left(1-\bar{u} / u_{e}\right) \mathrm{d} y$, respectively. Details about the spatial extent of the computational domains are identical to those in the fully adiabatic cases, see Wenzel et al. (2019), and the same holds for the numerical method.

\subsection{Simulation parameters}

As for the study in Wenzel et al. (2021), but in contrast to the adiabatic DNS results presented in Wenzel et al. (2019), the temperature at the solid wall is fixed according to a prescribed distribution $T_{w}=T_{w}(x)$. Except for this condition the numerical set-up of the non-adiabatic cases is identical to that of the adiabatic ones; see Wenzel et al. (2019). The reference thermodynamic flow properties are the inflow far-field temperature $T_{\infty, 0}=288.15 \mathrm{~K}$, inflow far-field density $\rho_{\infty, 0}=1.225 \mathrm{~kg} \mathrm{~m}^{-3}$, Prandtl number $P r=$ 0.71 , specific gas constant $R=287 \mathrm{~J} \mathrm{~kg}^{-1} \mathrm{~K}^{-1}$ and ratio of specific heats $\gamma=1.4$ and are set equal for all cases. Data averaging is performed over both time and the spanwise direction and does not start before the flow has passed the whole domain at least twice. Time averages were computed over a flow-through time corresponding to at least 250 local boundary-layer thicknesses $\delta_{99}\left(\Delta t u_{e} / \delta_{99}=250\right.$, where $\Delta t$ is the averaging period), which corresponds to at least 8 eddy-turnover times $\Delta t u_{\tau} / \delta_{99}$ for the most restricting APG cases. Both the appropriate convergence of the statistics and the initial transients have been assessed as described in Wenzel et al. (2018) and Wenzel et al. (2019). 


\section{Wenzel, T. Gibis and M. Kloker}

\section{Integral analysis - introduction and derivation}

Integral identities are valuable tools for the investigation of boundary-layer flows. By a wall-normal integration, mean-flow quantities varying in the wall-normal direction through the boundary layer are reduced to scalar quantities, allowing for a compact characterization of their streamwise evolution. Utilizing integral identities, momentum losses, for instance, can be assigned to different physical loss mechanisms, or they can help us understand and quantify flow-control strategies, among others. In the literature, integral relations have been obtained by integrating different types of balance laws such as momentum (von Kármán 1921; Fukagata et al. 2002) or different types of energy such as mean kinetic energy (Renard \& Deck 2016), mean exergy (Arntz, Atinault \& Merlen 2015) or mechanical energy (Drela 2009).

The main reason for the variety of identities is the difficulty in physically interpreting the results. In TBLs, for instance, the $\bar{\rho} u^{\prime \prime} v^{\prime \prime}$ stress is the most important stress increasing the shear stress in comparison with laminar flows, and thus should play a dominant role in the overall $c_{f}$ composition. In one of the simplest $c_{f}$ relations, namely the von Kármán momentum integral, however, the $\bar{\rho} \widetilde{u^{\prime \prime} v^{\prime \prime}}$ term does not contribute at all. Since the $\bar{\rho} \widetilde{u^{\prime \prime} v^{\prime \prime}}$ term simply redistributes momentum through turbulent mixing and feeds the viscous dissipation mechanisms close to the wall, its wall-normal integral is equal to zero (Deck et al. 2014):

$$
\int_{0}^{\delta_{e}} \frac{\partial \bar{\rho} \widetilde{u}^{\prime \prime} v^{\prime \prime}}{\partial y} \mathrm{~d} y=0
$$

This shows the difficulty of deriving integral relationships that actually reflect the relevance of important physical mechanisms.

In this study, the behaviour of both the $x$-momentum and the total-enthalpy equations are analysed by using integral relations for $c_{f}$ and $c_{h}$. To utilize essential aspects of the SRA, the contributing terms of the identities should remain formally very close to those of the $x$-momentum and the total-enthalpy equations. Moreover, a similar derivation procedure should be applicable to both equations. From this perspective, an integral approach comparable to the FIK identity is advantageous compared with other approaches such as the identity derived by Renard \& Deck (2016), henceforth referred to as the RD identity. After briefly introducing the basic idea of the classical FIK identity in the following, the integral identities used are presented.

\subsection{The FIK identity}

The FIK identity (Fukagata et al. (2002)) is based on a threefold repeated integration of the Navier-Stokes $x$-momentum equation in the wall-normal direction. This introduces weight functions like $\left(y_{b}-y\right)$ to terms such as the $\bar{\rho} u^{\prime \prime} v^{\prime \prime}$ stress,

$$
-\int_{0}^{y_{b}} \int_{0}^{y} \int_{0}^{y} \frac{\partial \bar{\rho} \widetilde{u}^{\prime \prime} v^{\prime \prime}}{\partial y} \mathrm{~d} y \mathrm{~d} y \mathrm{~d} y=-\int_{0}^{y_{b}}\left(y_{b}-y\right)\left[\bar{\rho} \widetilde{u^{\prime \prime} v^{\prime \prime}}\right] \mathrm{d} y \neq 0,
$$

where $y_{b}$ is the non-dimensionalized upper integration bound. These weights enhance the integrand near the wall, $\left.\left(y_{b}-y\right)\right|_{y=0}=y_{b}$, and reduce it in the outer region, $\left.\left(y_{b}-y\right)\right|_{y=y_{b}}=0$. According to its original formulation, the resulting terms of the FIK identity can be associated with four different skin-friction generating mechanisms $c_{f}=$ $c_{f}^{L}+c_{f}^{T}+c_{f}^{I}+c_{f}^{t}$, a laminar one $c_{f}^{L}$, a turbulent one $c_{f}^{T}$, an inhomogeneous one $c_{f}^{I}$ and a transient one $c_{f}^{t}$, of which the second is the weighted integral of the Reynolds shear-stress 
distribution (3.2); see Fukagata et al. (2002). In recent years, the incompressible FIK identity has been further developed and modified, such as by Mehdi \& White (2011), Mehdi et al. (2014), Peet \& Sagaut (2009), Bannier, Garnier \& Sagaut (2015), Yoon et al. (2016) and Modesti et al. (2018), and has become a common tool used in turbulent flow analysis and control; see e.g. Iwamoto et al. (2005), Kametani \& Fukagata (2011), Kametani et al. (2015) and Stroh et al. (2016). In contrast to the incompressible regime, only a few detailed studies are available for compressible flat-plate TBLs. In Gomez, Flutet \& Sagaut (2009), a possible compressible form of the FIK identity has been derived and corresponding effects on the mean skin-friction drag generation were studied, but without a full clarification of the compressibility effects on the various contributions as mentioned in Li et al. (2019).

It is a drawback of the FIK identity, however, that some of the contributing terms can easily be misinterpreted (especially for spatially evolving TBLs); see Deck et al. (2014), Renard \& Deck (2016) and Fan, Cheng \& Li (2019). One of the key controversies is that there is no simple justification for the threefold repeated integration and thus no physics-based explanation for the linearly weighted Reynolds shear stress (3.2). In the authors' opinion, the problem in justifying the threefold repeated integration is further evidenced by the fact that the threefold repeated integration is not necessary for deriving a valid integral identity for $c_{f}$. According to Cauchy's formula for repeated integration, $n$ repeated integrations of a continuous function can be rewritten into a single integral,

$$
f^{-n}\left(y_{b}\right)=\int_{y_{u}}^{y_{b}} \int_{y_{u}}^{\xi} \ldots \int_{y_{u}}^{\xi_{n-1}} f\left(\xi_{n}\right) \mathrm{d} \xi_{n} \ldots \mathrm{d} \xi_{2} \mathrm{~d} \xi_{1}=\frac{1}{(n-1) !} \int_{y_{u}}^{y_{b}}\left(y_{b}-y\right)^{n-1} f(y) \mathrm{d} y .
$$

This means that any number of repetitions greater than one would result in a FIK-like identity, since $c_{f}$ already appears as a variable in the equations after the first integration, as shown in Appendix A; variations in the number of repetitions essentially only affect the exponent of the respective weighting factors, as in (3.3).

In the authors' opinion, there are major advantages of using only a twofold repeated integration. For a clear argument, the resulting integral identities for $c_{f}$ and $c_{h}$ are introduced first, before this choice is justified in $\S 3.4$.

\subsection{The compressible integral identities for $c_{f}$ and $c_{h}$}

In this study, the same mix of Reynolds-averaged and Favre-averaged mean quantities is used as in Huang, Coleman \& Bradshaw (1995). The Favre decomposition is applied to the convective terms; for all other terms, the Reynolds-averaged decomposition is applied. To allow for a unique interpretation of the effects of Reynolds number, Mach number and heat transfer, the integral identities are presented in dimensionless form with $f=$ $\hat{f} / f_{n}$, where $\hat{f}$ is the dimensional quantity. Reference values for non-dimensionalization of $f_{n}$ are defined as $\rho_{n}=\rho_{e}, p_{n}=p_{e}, \mu_{n}=\bar{\mu}_{w}, u_{n}=u_{e}, L_{n}=\delta_{e}, T_{n}=\bar{T}_{r}-T_{w}$ and $h_{n}=$ $h_{0, n}=c_{p}\left(\bar{T}_{r}-T_{w}\right)$ to ensure the appropriate non-dimensionalization of $c_{f} \equiv \bar{\tau}_{w} /\left(\rho_{e} u_{e}^{2} / 2\right)$ and $c_{h} \equiv \bar{q}_{y, w} /\left(\rho_{e} u_{e} c_{p}\left(T_{w}-\bar{T}_{r}\right)\right)$. With $\tilde{h}_{0}=\tilde{h}+(E c / 2) \tilde{u}_{i} \tilde{u}_{i}+(E c / 2) \tilde{u}_{i}^{\prime \prime} u_{i}^{\prime \prime}$, and with the Reynolds number, Mach number and Eckert number defined as

$$
R e=\frac{\rho_{e} u_{e} \delta_{e}}{\bar{\mu}_{w}}, \quad M_{e}^{2}=\frac{\rho_{e} u_{e}^{2}}{\gamma p_{e}}, \quad E c=\frac{u_{e}^{2}}{c_{p}\left(\bar{T}_{r}-T_{w}\right)}=\frac{(\gamma-1) M_{e}^{2} T_{e}}{\left(\bar{T}_{r}-T_{w}\right)},
$$




\section{Wenzel, T. Gibis and M. Kloker}

the simplified two-dimensional (2-D) boundary-layer equations for the $x$-momentum and total-enthalpy can be written as

$$
\begin{aligned}
& \bar{\rho} \tilde{u} \frac{\partial \tilde{u}}{\partial x}+\bar{\rho} \tilde{v} \frac{\partial \tilde{u}}{\partial y}=-\frac{1}{\gamma M_{e}^{2}} \frac{\partial \bar{p}}{\partial x}+\frac{1}{R e}\left[\frac{\partial \bar{\tau}_{x x}}{\partial x}+\frac{\partial \bar{\tau}_{x y}}{\partial y}\right]-\frac{\partial \bar{\rho} u^{\prime \prime} u^{\prime \prime}}{\partial x}-\frac{\partial \bar{\rho} u^{\prime \prime} v^{\prime \prime}}{\partial y}, \\
& \bar{\rho} \tilde{u} \frac{\partial \tilde{h}_{0}}{\partial x}+\bar{\rho} \tilde{v} \frac{\partial \tilde{h}_{0}}{\partial y}=\frac{E c}{\operatorname{Re}}\left[\frac{\partial}{\partial y}\left(\bar{\tau}_{x y} \bar{u}+\bar{\tau}_{y y} \bar{v}\right)\right]+\frac{E c}{\operatorname{Re}}\left[\frac{\partial}{\partial y}\left(\overline{\tau_{x y}^{\prime} u^{\prime}}+\overline{\tau_{y y}^{\prime} v^{\prime}}+\overline{\tau_{z y}^{\prime} w^{\prime}}\right)\right] \\
& -\frac{\partial \bar{\rho} v^{\prime \prime} h^{\prime \prime}}{\partial y}-\frac{\partial \bar{\rho} u^{\prime \prime} h^{\prime \prime}}{\partial x}-\frac{E c}{2}\left[\frac{\partial}{\partial y}\left(\widetilde{\rho} \widetilde{u^{\prime \prime 2} v^{\prime \prime}}+\bar{\rho} \widetilde{v^{\prime \prime}}+\widetilde{\rho} \widetilde{w^{\prime \prime 2} v^{\prime \prime}}\right)\right] \\
& -E c\left[\frac{\partial}{\partial y}\left(\tilde{u} \bar{\rho} \widetilde{u}^{\prime \prime} v^{\prime \prime}+\tilde{v} \bar{\rho} v^{\prime \prime 2}\right)\right]-E c \frac{\partial \tilde{u} \bar{\rho} \tilde{u}^{\prime \prime 2}}{\partial x}-\frac{1}{\operatorname{RePr}} \frac{\partial \bar{q}_{y}}{\partial y} \text {. }
\end{aligned}
$$

\subsection{Integral identities}

Using the assumptions for quasi-2-D boundary-layer flow and a twofold repeated integration of (3.5), the integral identity for $c_{f}$ yields

$$
\begin{aligned}
& c_{f}=\underbrace{\frac{2}{\operatorname{Re} y_{b}} \int_{0}^{y_{b}}\left[\bar{\mu} \frac{\partial \bar{u}}{\partial y}\right] \mathrm{d} y}_{c_{f}^{L}}-\underbrace{\frac{2}{y_{b}} \int_{0}^{y_{b}}\left(y_{b}-y\right)\left[\tilde{v} \bar{\rho} \frac{\partial \tilde{u}}{\partial y}\right] \mathrm{d} y}_{c_{f}^{M}} \\
& \text { boundary-layer term mean-convection term } \\
& -\underbrace{\frac{2}{y_{b}} \int_{0}^{y_{b}}\left[\bar{\rho} \tilde{u}^{\prime \prime} v^{\prime \prime}\right] \mathrm{d} y}_{\begin{array}{c}
c_{f}^{T} \\
\text { turbulent-convection term }
\end{array}}+\underbrace{}_{\begin{array}{c}
c_{f}^{D} \\
y_{b}
\end{array} \int_{0}^{2} \int_{\text {spatial-development term }}^{y_{b}}\left(y_{b}-y\right)\left[-\tilde{u} \bar{\rho} \frac{\partial \tilde{u}}{\partial x}-\frac{1}{\gamma M_{e}^{2}} \frac{\partial \bar{p}}{\partial x}\right] \mathrm{d} y} .
\end{aligned}
$$

The non-dimensionalized upper integration bound $y_{b}$ becomes equal to one if the integration is performed up to the boundary-layer edge $\delta_{e}$; see also $\S 3.4$.

For the integral identity of the Stanton number $c_{h}$, a twofold repeated integration of the total-enthalpy equation in (3.6) results in

$$
\begin{aligned}
& c_{h}=\underbrace{\frac{1}{\operatorname{Re} y_{b}} \int_{0}^{y_{b}}\left[\frac{1}{\operatorname{Pr}} \bar{\mu} \frac{\partial \bar{T}}{\partial y}+E c \bar{u} \bar{\tau}_{x y}\right] \mathrm{d} y}_{c_{h}^{L}}-\underbrace{\frac{1}{y_{b}} \int_{0}^{y_{b}}\left(y_{b}-y\right)\left[\bar{\rho} \tilde{v} \frac{\partial \tilde{h}_{0}}{\partial y}\right] \mathrm{d} y}_{c_{h}^{M}} \\
& \text { boundary-layer term mean-convection term } \\
& -\underbrace{\frac{1}{y_{b}} \int_{0}^{y_{b}}\left[\bar{\rho} \widetilde{v}^{\pi \prime} h^{\prime \prime}+E c\left(\tilde{u} \bar{\rho} \widetilde{u^{\prime \prime} v^{\prime \prime}}+\tilde{v} \bar{\rho} \widetilde{v^{\prime \prime 2}}\right)+\frac{E c}{2}\left(\bar{\rho} \widetilde{u^{\prime \prime 2} v^{\prime \prime}}+\bar{\rho} \widetilde{v^{\prime \prime 3}}+\bar{\rho} \widetilde{w^{\prime \prime 2} v^{\prime \prime}}\right)\right] \mathrm{d} y}_{c_{h}^{T}} \\
& \text { turbulent-convection term }
\end{aligned}
$$




$$
-\underbrace{\text { spal-development term }}_{\begin{array}{c}
c_{h}^{D} \\
y_{b}
\end{array} \int_{0}^{y_{b}}\left(y_{b}-y\right)\left[\bar{\rho} \tilde{u} \frac{\partial \tilde{h}_{0}}{\partial x}+\frac{\partial \bar{\rho} \tilde{u}^{\prime \prime} h^{\prime \prime}}{\partial x}+E c \frac{\partial \tilde{u} \bar{\rho} \widetilde{u^{\prime \prime 2}}}{\partial x}\right] \mathrm{d} y}
$$

A detailed derivation of the $c_{f}$ and $c_{h}$ identities for an arbitrary number $n$ of repeated integrations is given in Appendix A. In contrast to the more complete forms of the identities in (A2) and (A4), both the viscous-stress fluctuation terms $c_{f}^{V F}$ and $c_{h}^{V F}$ are neglected in (3.7) and (3.8) for simplification, respectively. However, these terms have a noticeable share near the inflow regions of the simulation domain, where the flow is not yet fully turbulent. For the composition of $c_{h}$, for instance, $c_{h}^{V F}$ can contribute up to $30 \%$. For boundary layers rapidly changing in the streamwise direction, these terms therefore should be accounted for. For all figures shown hereafter, the inlet regions are cropped.

\subsection{Remarks}

The following remarks should be made about the benefits and limitations of the integral identities.

\subsubsection{General remarks on the FIK identity}

The first remark is related to the threefold repeated integration used in the original derivation of the FIK identity; see (3.2). As mentioned, the three integrations are not a requirement in deriving an integral condition for $c_{f}$ or $c_{h}$, since an arbitrary number of repeated integrations $n$ provides a valid decomposition of $c_{f}$ or $c_{h}$. Exemplarily evaluated for $n=2,3,4$ and 10 for the subsonic $i Z P G_{M=0.5}^{+10 \mathrm{~K}}$ case, the streamwise evolution of the integral identity (A2) is evaluated in Appendix B.2. Briefly, increasing the number of integrations $n$ increases the weights of terms that are dominant near the wall. Nevertheless, even though $n$ can be freely chosen from a mathematical point of view, not every choice of $n$ allows a physically plausible interpretation. In the original formulation of the FIK identity, the motivation for the threefold repeated integration is based on the viscous mean stress term, which reads in the dimensional formulation as

$$
\int_{0}^{y_{b}} \int_{0}^{y} \int_{0}^{y} \frac{\partial}{\partial y}\left(\bar{\mu} \frac{\partial \bar{u}}{\partial y}\right) \mathrm{d} y \mathrm{~d} y \mathrm{~d} y .
$$

By an appropriate choice, the viscosity can be normalized to one by introducing the Reynolds number into the dimensionless representation, and the integrand simplifies to $\partial / \partial y(\partial \bar{u} / \partial y)$. According to Fukagata et al. (2002), the first integration then results in a force balance, the second leads to the mean velocity profile and the third is akin to obtaining the flow rate. As discussed in Renard \& Deck (2016), however, this interpretation is only a consequence of the dimensionless formulation of the identity and therefore not intuitive, since the product of a force (first integral) and a length (due to the second integral) has the dimension of energy rather than velocity in dimensional representation.

From the authors' point of view, there are several reasons why using a twofold repeated integration is more appropriate. (i) From a physical point of view, the first integration gives a force/energy balance between the wall and all locations within the boundary layer. The second integration represents its average in the wall-normal direction. In other 


\section{Wenzel, T. Gibis and M. Kloker}

words, both integral identities simply quantify the wall-normal average of how much momentum/energy is transported towards the wall (positive) or away from the wall (negative) by each term. (ii) Compared with higher-fold integrations, the result is easier to interpret since all terms in the boundary-layer equations appearing with a derivative in the wall-normal direction are integrated without being weighted by a wall-distance-dependent term as in the classical FIK identity. In the present formulation chosen, this holds both for the $c_{f}^{T}$ and the $c_{f}^{L}$ terms in (3.7). The resulting values for $c_{f}^{L}$ and $c_{f}^{T}$ can be therefore simply associated with the area enclosed by the $\bar{\mu} \partial \bar{u} / \partial y$ and $\bar{\rho} u^{\prime \prime} v^{\prime \prime}$ distributions, respectively. Note that the main results derived below concerning the effects of pressure gradients, heat transfer and compressibility are qualitatively identical for $n=2$ and $n=3$.

\subsubsection{Remarks on the upper integration limit}

Regardless of the upper integration limit $y_{b}$, both identities yield a valid decomposition of $c_{f}$ and $c_{h}$. However, the choice of $y_{b}$ greatly influences the portions of the individual terms with respect to each other. Usually, both the upper integration limit and the reference length for non-dimensionalization are set to $\delta_{99}$, which simplifies $y_{d}$ to one in (3.7) and (3.8). An exception is provided by the study of Mehdi et al. (2014), in which the influence of the lower and upper integration limits on the accuracy of the $c_{f}$ calculation is evaluated. For the compressible boundary layers in the present study, it seems reasonable to choose an upper integration bound of $y_{b}=\delta_{e} \approx 1.3 \delta_{99}$ to cover the thermal boundary layer. As this is quite an arbitrary choice, $y_{b}$ has been kept as a variable in the derivation of both identities; its influence on the resulting contributions is further discussed in Appendix B.3. In summary, a lower integration bound $y_{b}$ crops terms at $y_{b}$, thus increasing the portion of near-wall dominant terms like the $c_{f}^{L}$ term. A higher integration bound decreases the percentage share of near-wall dominant terms. Note that - as long as $y_{b}$ remains at approximately 1 - the particular choice of $y_{b}$ does not affect the qualitative conclusions drawn in the following.

\subsubsection{Remarks on the splitting of terms}

Variants of the original FIK identity are found in the literature, modified for specific applications. For the original purpose of understanding flow-control strategies and drag-reducing mechanisms, terms are often separated into different components of interest. In experimental studies, where often only the wall-normal profiles are available, also all $x$-derivatives are usually replaced with $y$-derivatives to allow for an accurate evaluation. For the formulations chosen in the present study, however, individual components of the momentum and total-enthalpy equations are intended to be easily recognizable in a consistent form. Hence, a further subdivision of the individual terms is omitted. Furthermore, the continuity equation has been used to reformulate the mean-convection terms to avoid large, counteracting components that essentially sum to zero.

\section{Streamwise evolution of $c_{f}$ and $c_{h}$}

In the following sections the integral identities for $c_{f}$ and $c_{h}$ are evaluated. Since all results can be simultaneously affected by compressibility, heat transfer and the pressure gradient, meaningful conclusions can only be derived if the trends observed are isolated and clearly associated with the variation of only one of the influencing parameters. To allow for a better understanding of the raw data used, the spatial evolutions of the integral identities 


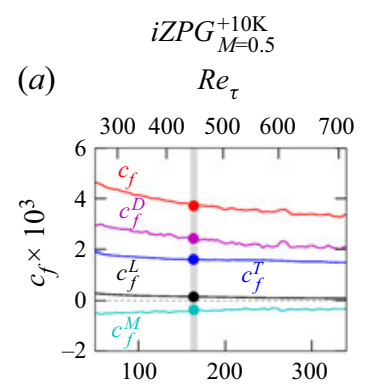

(b) $\quad{ }^{i A P G_{\beta_{K}}=0.6}$

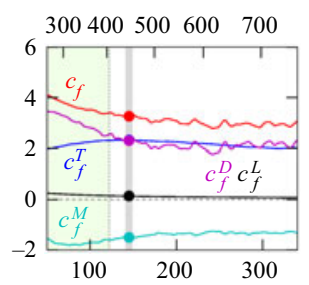

(e) $\quad 300400500 \quad 600 \quad 700$

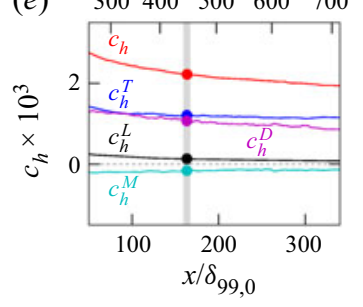

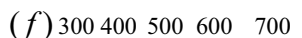

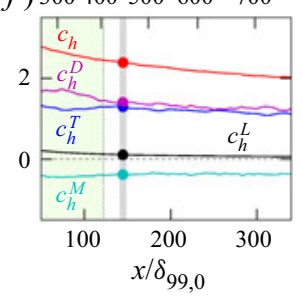

(c)
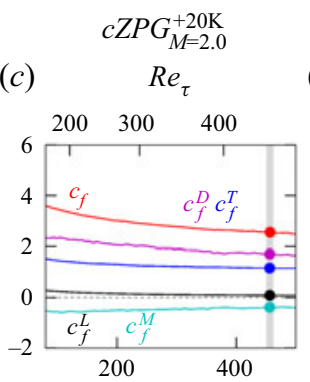

(g) $200 \quad 300 \quad 400$

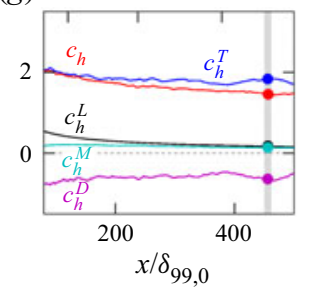

(d)
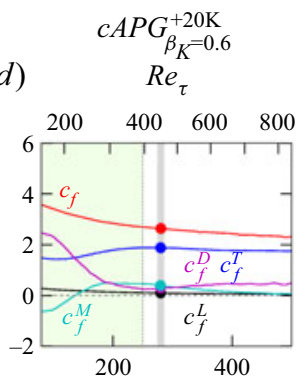

(h) $200 \quad 400 \quad 600 \quad 800$

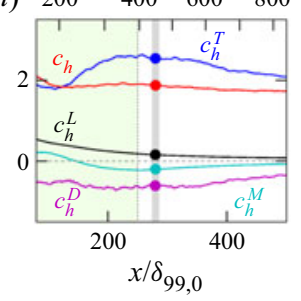

Figure 1. Evaluation of the $c_{f}$ identity (3.7) in panels $(a-d)$ and the $c_{h}$ identity (3.8) in panels $(e-h)$; depicted are the subsonic $i Z P G_{M=0.5}^{+10 \mathrm{~K}}$ and the $i A P G_{\beta_{K}=0.6}^{10 \mathrm{~K}}$ cases on the left-hand side and the supersonic $c Z P G_{M=2.0}^{+20 \mathrm{~K}}$ and $c A P G_{\beta_{K}=0.6}^{+20 \mathrm{~K}}$ cases on the right-hand side. Values at $R e_{\tau}=450$ are marked for a visual comparison. Green regions in panels $(b, d, f, h)$ represent regions where the APG cases are not yet self-similar.

for $c_{f}$ and $c_{h}$ are described qualitatively in figure 1 first. Here the focus is on working out relevant trends, on which the detailed investigations in $\S \S 5$ and 6 are built. Depicted are the subsonic, nearly incompressible $i Z P G_{M=0.5}^{+10 \mathrm{~K}}$ and $i A P G_{\beta_{K}=0.6}^{+10 \mathrm{~K}}$ cases on the left-hand side and the supersonic $c Z P G_{M=2.0}^{+20 \mathrm{~K}}$ and $c A P G_{\beta_{K}=0.6}^{+20 \mathrm{~K}}$ cases on the right-hand side. The lower abscissa gives the spatial position in the domain normalized with the boundary-layer thickness at the inlet of the domain $x / \delta_{99,0}\left(\delta_{99,0}\right.$ is equal for all cases), and the upper abscissa gives the corresponding Reynolds number $R e_{\tau}$. All contributing terms $c_{(f, h)}^{L}$, $c_{(f, h)}^{T}, c_{(f, h)}^{M}$ and $c_{(f, h)}^{D}$ are represented by coloured lines, with their sum as a red line. Values at $R e_{\tau}=450$ are marked for comparison, and regions where the APG cases are not yet self-similar are shaded in green. For both $c_{f}$ and $c_{h}$, the constituent components of the individual terms $c_{h}^{L}=\sum_{i=1}^{2} c_{h}^{L, i}, c_{h}^{T}=\sum_{i=1}^{6} c_{h}^{T, i}, c_{f}^{D}=\sum_{i=1}^{2} c_{f}^{D, i}$ and $c_{h}^{D}=\sum_{i=1}^{3} c_{h}^{D, i}$ are depicted in Appendix B.4 in figures 13 and 14, respectively, without being discussed in detail.

\subsection{Streamwise evolution of $c_{f}$}

According to (3.7), the $c_{f}$ distributions depicted in figure $1(a-d)$ are dominated by $c_{f}^{T}$, $c_{f}^{M}$ and $c_{f}^{D} ; c_{f}^{L}$ only provides a small portion of the overall $c_{f}$ (especially for high $R e$; see e.g. Fan et al. 2020). For both the sub- and supersonic ZPG cases in figure 1(a,c), respectively, the spatial-development term $c_{f}^{D}$ constitutes the largest portion. For the suband supersonic APG cases in figure $1(b, d)$, the $c_{f}^{D}$ distributions are influenced in a different manner by the pressure gradient in regions of approximated self-similarity, increased in panel $(b)$ and strongly decreased in panel $(d)$. The mean-convection term $c_{f}^{M}$ essentially balances the changes in $c_{f}^{D}$. A comparison of the turbulence terms $c_{f}^{T}$ (blue) reveals almost 


\section{Wenzel, T. Gibis and M. Kloker}

identical behaviour for the sub- and supersonic ZPG cases in panels $(a, c)$ and the sub- and supersonic APG cases in panels $(b, d)$, respectively. The percentage contribution of $c_{f}^{T}$ is therefore expected to be predominantly influenced by the pressure-gradient parameter $\beta_{K}$, and only marginally by the local Mach number.

\subsection{Streamwise evolution of $c_{h}$}

According to (3.8), the results of the $c_{h}$ distributions are depicted in figure $1(e-h)$. For two reasons, the results are more complex to describe than for the $c_{f}$ distributions. First, the total enthalpy describes the overall change in enthalpy, mean kinetic energy and mean turbulent kinetic energy. Hence, more terms have to be taken into account to capture a complete representation of $c_{h}$. Second, the $c_{h}$ distributions are strongly influenced by the pressure-gradient parameter $\beta_{K}$, the local Mach number and heat transfer. For the subsonic $i Z P G_{M=0.5}^{+10 \mathrm{~K}}$ case in figure 1(e), comparable trends can be found to those for the $c_{f}$ contributions in panel (a); only the relative proportions of $c_{h}^{T}$ and $c_{h}^{D}$ differ. A comparison between the subsonic $i Z P G_{M=0.5}^{+10 \mathrm{~K}}$ and $i A P G_{\beta_{K}=0.6}^{+10 \mathrm{~K}}$ cases in panels $(e, f)$, respectively, shows only a small influence of the streamwise pressure gradient. For the supersonic cases in panels $(g, h)$, the blue $c_{h}^{T}$ contributions are noticeably increased in comparison with the subsonic cases. For the supersonic $c Z P G_{M=2.0}^{+20 \mathrm{~K}}$ case in panel $(g), c_{h}^{T}$ is increased from approximately $60 \%$ of $c_{h}$ to approximately $120 \%$ of $c_{h}$ at $R e_{\tau}=450$, and for the supersonic $c A P G_{\beta_{K}=0.6}^{+20 \mathrm{~K}}$ case in $(h)$ to approximately $140 \%$ of $c_{h}$. The increase in $c_{h}^{T}$ is mainly balanced by a decrease of the spatial evolution term $c_{h}^{D}$ to negative values. Note that, in addition to the overall terms $c_{h}^{L}, c_{h}^{M}, c_{h}^{T}$ and $c_{h}^{D}$ shown here, the portions of the individual terms also show a complex behaviour; see figure 14 .

\section{Detailed evaluation of the $c_{f}$ identity}

This section's primary goal is to clearly assign the previously observed variations in the $c_{f}$ identity to the physical effects of compressibility, heat transfer and pressure gradient. In figure $1(a-d)$ it was shown that the pressure-gradient parameter $\beta_{K}$ is the decisive influencing parameter for the $c_{f}$ identity. Consequently, all results are plotted against their local $\beta_{K}$ value in this section. This approach isolates the main influencing factor and allows an evaluation of how much the other factors, compressibility and heat transfer, affect the portions of each component in the $c_{f}$ identity.

Normalized with $c_{f}$ and $2 c_{h}$, respectively, the ratios of the single components $c_{f}^{x} / c_{f}$ are depicted in figure $2(a)$ and the ratios of $c_{f}^{x} /\left(2 c_{h}\right)$ in figure $2(b)$. The corresponding $c_{f}$ and $c_{h}$ distributions are depicted in Appendix B.1 in figure 10. In figure 2(a,b) results are plotted as dots with transparent colour for every 20th data point in the streamwise direction in regions of approximate self-similarity. Regions near the inlet of the computational domain, where the streamwise pressure gradient is slowly imposed and the resulting flow is not yet self-similar, are depicted as thick, transparent lines for each case. Since the mean-convection term $c_{f}^{M}$ and the spatial evolution term $c_{f}^{D}$ exhibit counteracting behaviour, the two will be considered as one term $\left(c_{f}^{M}+c_{f}^{D}\right)$ hereafter.

\subsection{Evaluation of $c_{f}^{x} / c_{f}$}

First the ratio $c_{f}^{x} / c_{f}$ in panel $(a)$ is discussed, quantifying the portion of every component $c_{f}^{x}$ in the overall $c_{f}$. A comparison of the various Mach-number cases shows almost identical 

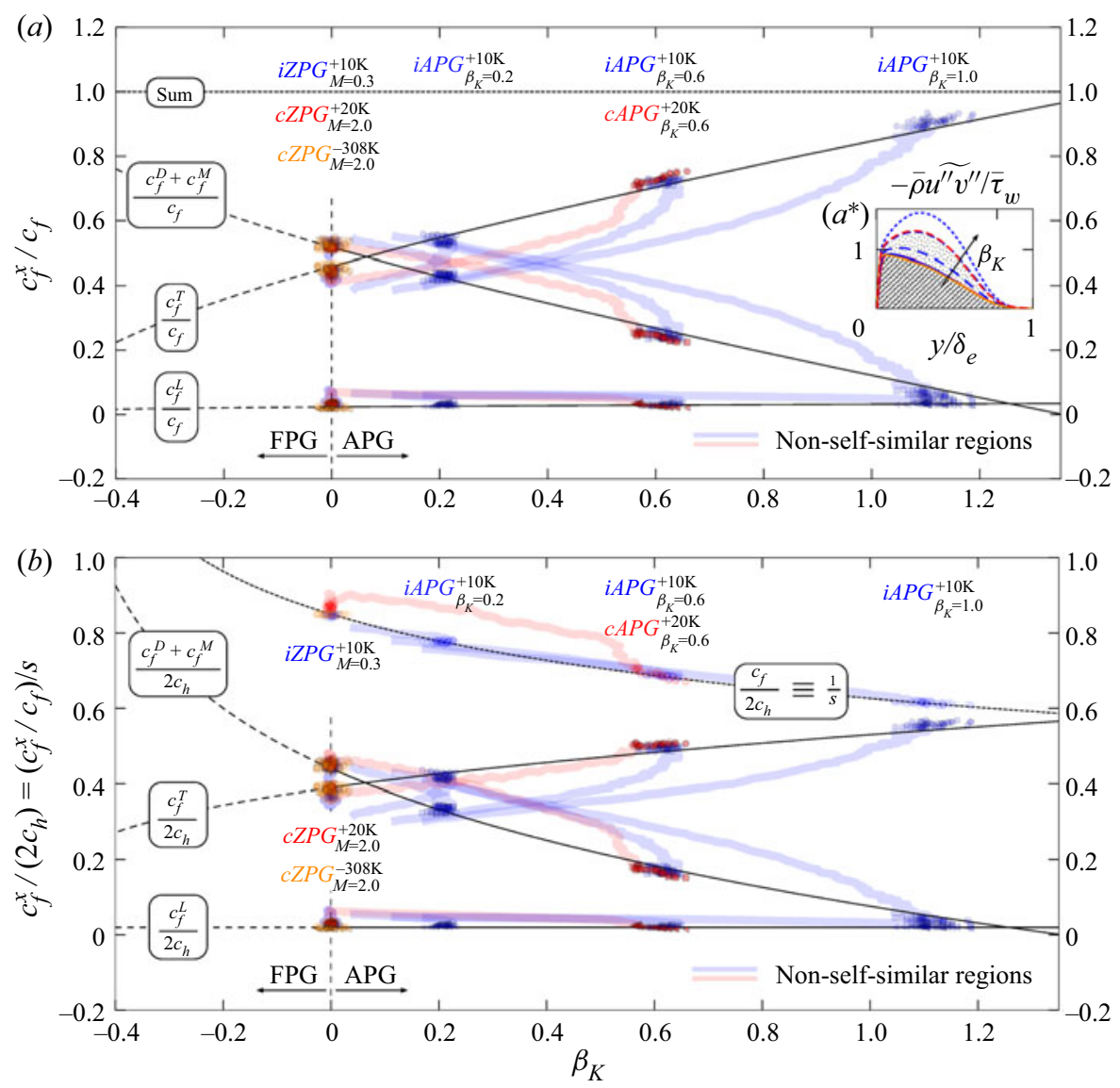

Figure 2. Evaluation of the $c_{f}$ identity according to (3.7) as function of the kinematic Rotta-Clauser parameter $\beta_{K}$. The ratio of $c_{f}^{x} / c_{f}$ is given in panel $(a)$, of $c_{f}^{x} /\left(2 c_{h}\right)$ in panel $(b)$. Symbols represent results evaluated at every 20th numerical grid point in the streamwise direction in regions of self-similarity. Subsonic cases are coloured red, supersonic cases blue and the strongly cooled supersonic case orange.

behaviour for the blue subsonic and the red supersonic cases. Hence, to allow for a simple argument, the influence of the pressure gradient is discussed based on the subsonic cases first, and then the implications of the Mach number/heat transfer are examined.

All in all, the blue subsonic results depicted in panel $(a)$ reflect the trends expected from figure 1 . The boundary-layer term $c_{f}^{L}$ is only slightly influenced by the pressure gradient's strength and contributes only approximately $2 \%-3 \%$ to $c_{f}$. For ZPG cases at $\beta_{K}=0$, the turbulent-convection term $c_{f}^{T}$ contributes approximately $44 \%$ to $c_{f}$. For increasing APG strength this portion increases steeply, while an extrapolation for favourable-pressure-gradient cases $\left(\beta_{K}<0\right)$ suggests a decrease. The sum of $c_{f}^{D}$ and $c_{f}^{M}$ constitutes the remaining part and thus essentially behaves in a contrary manner to $c_{f}^{T}$ with respect to $\beta_{K}$. In principle, the explanation of the trends shown is straightforward: the normalization of the individual components $c_{f}^{x}$ with $c_{f}$ essentially corresponds to a normalization with the wall shear stress $\bar{\tau}_{w}$ (inner scaling). Hence, the share of $c_{f}^{L} / c_{f}$ corresponds to the average value of $(\bar{\mu} \partial \bar{u} / \partial y) / \bar{\tau}_{w}$ between $0 \leq y \leq y_{b}$, and the share of 


\section{Wenzel, T. Gibis and M. Kloker}

$c_{f}^{T} / c_{f}$ to the average value of $\left(\bar{\rho} u^{\prime \prime} v^{\prime \prime}\right) / \bar{\tau}_{w}$. As known, the turbulence term $\left(\bar{\rho} \widetilde{u}^{\prime \prime} v^{\prime \prime}\right) / \bar{\tau}_{w}$ increases considerably for APG cases compared with ZPG cases, explaining the increasing share of $c_{f}^{T} / c_{f}$ for increasing $\beta_{K}$. As shown in Wenzel et al. (2019), the distributions of $(\bar{\mu} \partial \bar{u} / \partial y) / \bar{\tau}_{w}$ are almost unaffected by $\beta_{K}$.

Next, the effects of Mach number and heat transfer are assessed. Indicated by the clustering of both sub- and supersonic cases, only a small influence of the local Mach number is visible in panel $(a)$. According to Morkovin's transformation, the distributions of $\bar{\rho} u^{\prime \prime} v^{\prime \prime} / \bar{\tau}_{w}$ - and hence also its integral version $c_{f}^{T} / c_{f}$ - are Mach-number invariant; compare the good agreement of the sub- and supersonic cases (coloured blue and red) in panels $(a)$ and $\left(a^{*}\right)$ for cases with the same $\beta_{K}$. To assess the influence of heat transfer, a comparison between the supersonic near-adiabatic $c Z P G_{M=2.0}^{+20 \mathrm{~K}}$ case (in red) and the strongly cooled supersonic $Z P G_{M=2.0}^{-308 \mathrm{~K}}$ case (in orange) does not reveal a recognizable influence of the wall temperature. The same holds for the near-adiabatic pressure-gradient cases; however, it cannot be excluded that this changes for strongly heated or cooled conditions. In summary, the influences of Mach number and heat transfer can be seen to act comparably on the individual $c_{f}^{x}$ terms for the cases considered. This causes their relative contributions to the overall $c_{f}$ always to remain approximately the same, even if $c_{f}$ changes significantly; compare the $c_{f}$ and $c_{h}$ distributions given in figure 10 .

For the remaining part of this study, it is useful to approximate the shown trends as a function of the local pressure gradient $\beta_{K}$. As it is simpler than a direct dependence on $\beta_{K}$, an empirical approximation as a function of the Reynolds analogy factor $s=f\left(\beta_{K}, \ldots\right)$ is used:

$$
\frac{c_{f}^{T}}{c_{f}} \approx 0.3315 s^{2}, \quad \frac{c_{f}^{L}}{c_{f}} \approx 0.02 s, \quad \frac{c_{f}^{D}+c_{f}^{M}}{c_{f}} \approx 1-0.3315 s^{2}-0.02 s
$$

see the solid black lines in panel (a). As introduced in more detail in Wenzel et al. (2021), the distribution for $s$ is determined from the analytically derived relation by So (1994), which is based on classical, incompressible self-similarity assumptions; see also panel (b):

$$
s=\frac{2 c_{h}}{c_{f}}=\frac{\kappa^{-1} \ln \left(\operatorname{Re}_{\delta_{K, w}^{*}}\right)+B+A\left(\beta_{K}\right)}{\kappa_{\theta}^{-1} \ln \left(R e_{\delta_{K, w}^{*}}^{*}\right)+B_{\theta}+A_{\theta}\left(\beta_{K}, P r_{t}\right)},
$$

where $\operatorname{Re}_{\delta_{K, w}^{*}}^{*}=16000, \kappa_{\theta}=0.41 / \operatorname{Pr}_{t}, B=4.9$ and $B_{\theta}=3.8$. Both $A\left(\beta_{K}\right)$ and $A_{\theta}\left(\beta_{K}, P r_{t}\right)$ are obtained from the solutions of differential equations for the wall-normal velocity and temperature profiles; see Mellor \& Gibson (1966), So (1994) and Wenzel et al. (2021) for details. The depicted approximations further allow a qualitative assessment of the non-self-similar regions of flow represented by the transparent lines in figure 2(a). Both the $c_{f}^{T}$ and $\left(c_{f}^{D}+c_{f}^{M}\right)$ terms deviate notably from the approximation curves for self-similar regions, for both sub- and supersonic cases. In APG regions, the turbulent contribution $c_{f}^{T}$ has a delayed reaction to the pressure gradient compared with a self-similar case at the same $\beta_{K}$; the sum of $\left(c_{f}^{D}+c_{f}^{M}\right)$ reacts prematurely. A closer look into the history effects in non-self-similar incompressible TBLs can be found in Tanarro, Vinuesa \& Schlatter (2020). 


\subsection{Evaluation of $c_{f}^{x} /\left(2 c_{h}\right)$}

Next, the results in figure $2(b)$ are discussed. With $c_{f}^{x} /\left(2 c_{h}\right)=\left(c_{f}^{x} / c_{f}\right) / s$, panel $(b)$ essentially represents a scaling of the $c_{f}^{x} / c_{f}$ distributions depicted in panel $(a)$ with $s$, and hence the approximations $(5.1 a-c)$ become

$$
\frac{c_{f}^{T}}{2 c_{h}} \approx 0.3315 s, \quad \frac{c_{f}^{L}}{2 c_{h}} \approx 0.02, \quad \frac{c_{f}^{D}+c_{f}^{M}}{2 c_{h}} \approx \frac{1}{s}-0.3315 s-0.02 ;
$$

compare the black solid lines in panel $(b)$. As the sum of all contributions results in the inverse of the local Reynolds analogy factor $1 / s=c_{f} /\left(2 c_{h}\right)$ as depicted in Wenzel et al. (2021), the individual portions $c_{f}^{x} /\left(2 c_{h}\right)$ quantify their relative contributions to $1 / s$.

As shown in panel $(a)$, the normalized contributions $c_{f}^{x} / c_{f}$ correspond to the integral version of Morkovin's transformation and therefore are almost Mach-number invariant. Since the Reynolds analogy factor $s$ is almost Mach-number invariant as well, this also holds for the components $c_{f}^{x} /\left(2 c_{h}\right)=\left(c_{f}^{x} / c_{f}\right) / s$. Therefore, in self-similar regions, the deviations of the single $c_{f}^{x}$ terms from the black approximations are nearly independent of whether they are scaled with $c_{f}$ as in panel $(a)$ or with $2 c_{h}$ as in panel $(b)$.

Concluding from panel $(b)$, the strong pressure-gradient dependence of the inverse of the Reynolds analogy factor $1 / s$ (or of $s$ as shown in Wenzel et al. 2021) can be attributed to the fact that the $c_{f}^{T} /\left(2 c_{h}\right)$ component increases more slowly with APGs than the $\left(c_{f}^{D}+c_{f}^{M}\right) /\left(2 c_{h}\right)$ component decreases. For incompressible cases, this reasoning is straightforward as $c_{f}$ is more affected by pressure gradients than $c_{h}$, making the variations of the $c_{f}^{x}$ contributions the driving factor of the variation in $s$. For the compressible cases, in contrast, the influence of $\beta_{K}$ on $c_{h}$ also can be stronger than on $c_{f}$, making this result $a$ priori not obvious; compare Wenzel et al. (2021).

Finally, if the non-self-similar flow regions are assessed for the subsonic cases, the blue transparent lines for $1 / \mathrm{s}$ closely follow the black dotted reference curve for self-similar cases. This indicates a synchronous response of both $c_{f}$ and $c_{h}$ on the imposed pressure gradient also in regions of non-self-similar flows, where the individual contributions $c_{f}^{x} / c_{f}$ clearly have not yet converged to a self-similar state. For the supersonic cases (red transparent lines), in contrast, the values for $1 / s$ are higher than the settled values in regions of non-self-similar flows, implying an asynchronous response of $c_{f}$ and $c_{h}$ to the imposed pressure gradient; see also Wenzel et al. (2021).

\section{Detailed evaluation of the $c_{h}$ identity}

In contrast to the $c_{f}^{x}$ components of the $c_{f}$ identity, the $c_{h}^{x}$ components of the $c_{h}$ identity are affected by all three influencing parameters; see $\S 4.2$. The following analysis is based on the following assumption/consideration: in the non-dimensionalized form of the total-enthalpy equation chosen, the Eckert number $E c=u_{e}^{2} /\left(c_{p}\left(\bar{T}_{r}-T_{w}\right)\right)=(\gamma-$ 1) $M_{e}^{2} T_{e} /\left(\bar{T}_{r}-T_{w}\right)$ appears as a relevant parameter accounting for temperature effects. Note that this definition differs from the very basic one (in gas dynamics) where just $T_{e}$ is used in the denominator (and thus $E c=(\gamma-1) M_{e}^{2}$ ), and from the one commonly used for heat-transfer investigations, where $\left(T_{w}-T_{e}\right)$ is used in the denominator. Since the $E c$ number incorporates both the Mach number and the wall temperature in the specific dimensionless form chosen, it appears to be a suitable parameter to commonly account for both effects. Its use essentially implies that the same integral behaviour is expected for flow cases with the same $E c$, regardless of whether a variation of $E c$ is caused by a change 
(a)

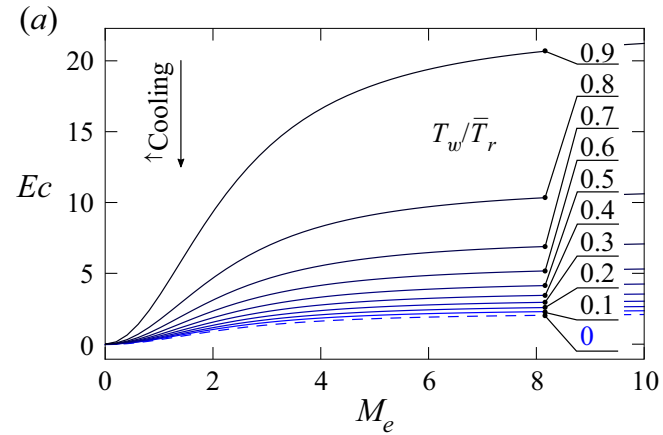

(b)

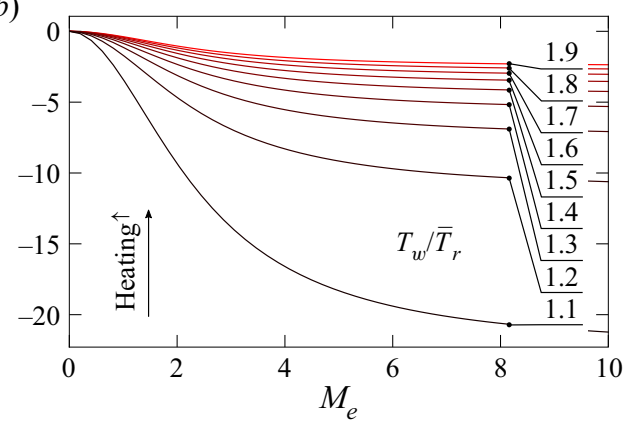

Figure 3. Evaluation of the Eckert number $E c=(\gamma-1) M_{e}^{2} T_{e} /\left(\bar{T}_{r}-T_{w}\right)$ as a function of $M_{e}$ and $T_{w} / \bar{T}_{r}$, if $\bar{T}_{r} / T_{e}=1+r(\gamma-1) / 2 M_{e}^{2}$ is assumed for ZPG cases. Cooled cases are depicted in panel $(a)$ and heated cases in panel $(b)$ for $\gamma=1.4$ and $r=\operatorname{Pr}^{1 / 3}$ with $\operatorname{Pr}=0.71$.

of the Mach number or of the wall temperature. This is in line with Morkovin's hypothesis implying that Mach number effects act comparably to heated walls for large regions of the TBL.

To get an idea of the $E c$-number behaviour in the parameter space, its dependence on both the local Mach number $M_{e}$ and the wall-to-recovery temperature ratio $T_{w} / \bar{T}_{r}$ is depicted in figure 3(a,b) for cooled and heated cases, respectively, if both $\gamma$ and $r$ are assumed constant. Both the heated and the cooled curves show the same behaviour, only differing in the $E c$ number's sign. As depicted, both increased heating and cooling as well as a reduction of $M_{e}$ reduces the absolute $E c$ number. Note that it becomes impossible to reach arbitrarily low positive $E c$ numbers for cooled cases and all Mach numbers in panel $(a)$, e.g. reaching $E c \lesssim 1$ at $M_{e}=2$. For very high Mach numbers, $E c$ becomes merely a function of $T_{w} / \bar{T}_{r}$ (and variations in $r$ if pressure gradients are applied; see Wenzel et al. 2019). Further, with increasing Mach number $M_{e}$, an increasingly strong heating or cooling $\left(\bar{T}_{r}-T_{w}\right)$ is necessary to achieve a low $E c$ number.

\subsection{Turbulent contribution $c_{h}^{T}$}

For understanding of the integral composition of $c_{h}$, see (3.8), the turbulent-convection term $c_{h}^{T}$ is of central importance and will be considered first:

$$
\begin{aligned}
& c_{h}^{T}=\underbrace{-\frac{1}{y_{b}} \int_{0}^{y_{b}}\left[\bar{\rho} v^{\prime \prime} h^{\prime \prime}\right] \mathrm{d} y}_{c_{h}^{T, 1}} \underbrace{-\frac{1}{y_{b}} \int_{0}^{y_{b}}\left[E c\left(\tilde{u} \bar{\rho} \widetilde{u}^{\prime \prime} v^{\prime \prime}+\tilde{v} \bar{\rho} v^{\prime \prime 2}\right)\right] \mathrm{d} y}_{c_{h}^{T, 2}} \\
& \underbrace{-\frac{1}{y_{b}} \int_{0}^{y_{b}}\left[\frac{E c}{2}\left(\bar{\rho} \widetilde{u^{\prime \prime 2} v^{\prime \prime}}+\bar{\rho} \widetilde{v^{\prime \prime 3}}+\widetilde{\rho} \widetilde{w^{\prime 2} v^{\prime \prime}}\right)\right] \mathrm{d} y .}_{c_{h}^{T, 3}}
\end{aligned}
$$

The enthalpy fluctuations are denoted by $c_{h}^{T, 1}$ and the fluctuations of the mean kinetic and turbulent kinetic energy (triple fluctuations) by $c_{h}^{T, 2}$ and $c_{h}^{T, 3}$, respectively. Following the idea that the SRA essentially describes the analogy between enthalpy transfer and 
kinetic-energy transfer, both kinetic-energy terms $c_{h}^{T, 2}$ and $c_{h}^{T, 3}$ are mainly considered as only a single term $c_{h}^{T, 2+3}$ in the following.

\subsubsection{Implications from the SRA}

Since the individual terms of the derived integral identities stay formally close to the terms of the momentum and total-enthalpy equations, implications derived from the SRA (Morkovin relations) can be directly transferred to the turbulence terms of the integral identity of $c_{h}$. Thus, by comparison with the DNS data, the validity of selected implications of the SRA can be evaluated from an integral perspective, depending on where a selected case is located in the parameter space considered.

As introduced in $\S 1$, the SRA assumes a direct proportionality between the streamwise velocity fluctuations $u^{\prime \prime}$ and the total-enthalpy fluctuations $h_{0}^{\prime \prime}$ according to

$$
E c k_{1} u^{\prime \prime} \approx h_{0}^{\prime \prime}=h^{\prime \prime}+E c \tilde{u}_{i} u_{i}^{\prime \prime}+\frac{E c}{2} \widetilde{u_{i}^{\prime \prime} u_{i}^{\prime \prime},}
$$

where $k_{1}=-1 /(\operatorname{Pr} E c)\left(\bar{q}_{w} / \bar{\tau}_{w}\right)$ takes into account wall heat fluxes in the case of non-adiabatic wall conditions. Multiplication by $\bar{\rho} v^{\prime \prime}$, time averaging and rearrangement yields

$$
\bar{\rho} v^{\prime \prime} h^{\prime \prime} \approx-E c\left(\tilde{u}_{i} \bar{\rho} \widetilde{u_{i}^{\prime \prime} v^{\prime \prime}}+\frac{1}{2} \bar{\rho} \widetilde{u_{i}^{\prime \prime 2} v^{\prime \prime}}\right)-\frac{1}{\operatorname{Pr}} \frac{\bar{q}_{w}}{\bar{\tau}_{w}} \bar{\rho} \widetilde{u^{\prime \prime} v^{\prime \prime}},
$$

which finally predicts the ratio between enthalpy-associated and kinetic-energy-associated fluctuations to be

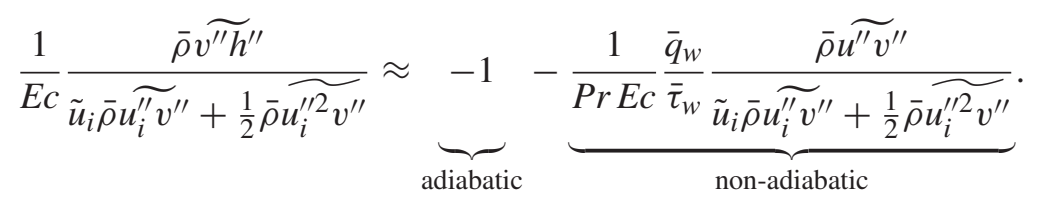

In terms of the integral identity, (6.3) becomes

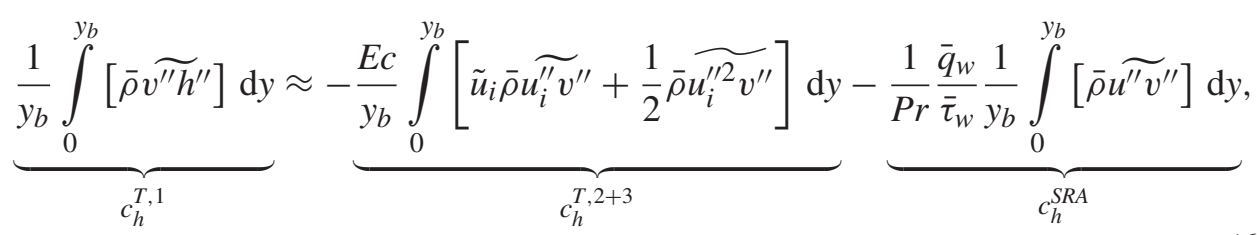

where the term including the wall heat flux $\bar{q}_{w}$ is denoted by $c_{h}^{S R A}$. Hence, the turbulent heat-flux term $c_{h}^{T, 1}$ predicted by the SRA can be considered as the sum of a non-adiabatic contribution $c_{h}^{S R A}$ and the contribution of the kinetic-energy fluctuations $c_{h}^{T, 2+3}$. As the $c_{h}^{S R A}$ term becomes zero for adiabatic flows, $c_{h}^{T, 2+3}$ is associated with the adiabatic portion of $c_{h}^{T, 1}$. Division of (6.5) by $c_{h}^{T, 2+3}$ finally yields the integral version of (6.4),

$$
\frac{c_{h}^{T, 1}}{c_{h}^{T, 2+3}} \approx \underbrace{-1}_{\text {adiabatic }}-\underbrace{\frac{c_{h}^{S R A}}{c_{h}^{T, 2+3}}}_{\text {non-adiabatic }}
$$




\section{Wenzel, T. Gibis and M. Kloker}

Remarks on the functional forms of $c_{h}^{T, 1}, c_{h}^{T, 2+3}$ and $c_{h}^{S R A}$. Below, the turbulent terms $c_{h}^{T, 1}, c_{h}^{T, 2+3}$ and $c_{h}^{S R A}$ are evaluated normalized by $c_{h}$, allowing for a rough estimation about their functional form. As the wall-normal integrals of both $\bar{\rho} u^{\prime \prime} v^{\prime \prime} / \bar{\tau}_{w}$ and the non-dimensionalized $\tilde{u}=\hat{\tilde{u}} / u_{e}$ only have a weak $E c$-number dependence in the case of self-similar flows, the normalized contributions $2 c_{h}^{T, 2+3} / c_{f}$ and hence $c_{h}^{T, 2+3} / c_{h}$ suggest an almost linear $E c$-number dependence of the form

$$
\frac{c_{h}^{T, 2+3}}{c_{h}} \approx C_{1}\left(\beta_{K}\right) E c .
$$

Here, $C_{1}\left(\beta_{K}\right)$ denotes a constant mainly depending on the pressure gradient's strength applied. With the same argument for the integrand of $c_{h}^{S R A}$ and the additional assumption that the ratio $\bar{q}_{w} / \bar{\tau}_{w}$ essentially resembles the $E c$-number-independent Reynolds analogy factor $s$, the course of $c_{h}^{S R A} / c_{h}$ is expected to behave as a constant $C_{2}\left(\beta_{K}\right)$ only depending on the local pressure-gradient strength,

$$
\frac{c_{h}^{S R A}}{c_{h}} \approx-C_{2}\left(\beta_{K}\right) .
$$

Using (6.5) and the modelling approaches (6.7) and (6.8), the $c_{h}^{T, 1} / c_{h}$ term should depend linearly on the $E c$ number:

$$
\frac{c_{h}^{T, 1}}{c_{h}} \approx C_{2}\left(\beta_{K}\right)-C_{1}\left(\beta_{K}\right) E c .
$$

Finally, with (6.7) and (6.9), the ratio $c_{h}^{T, 1} / c_{h}^{T, 2+3}$ should behave according to

$$
\frac{c_{h}^{T, 1}}{c_{h}^{T, 2+3}} \approx-1+\frac{1}{E c} \frac{C_{2}\left(\beta_{K}\right)}{C_{1}\left(\beta_{K}\right)} .
$$

Thus, the SRA predicts the ratio between the enthalpy and kinetic-energy fluctuations $c_{h}^{T, 1} / c_{h}^{T, 2+3}$ to behave like a hyperbola: for adiabatic conditions $(E c \rightarrow \pm \infty)$, it predicts a ratio of $c_{h}^{T, 1} / c_{h}^{T, 2+3} \approx-1$; for strongly heated or cooled cases $(E c \rightarrow \pm 0)$, it predicts a ratio of $c_{h}^{T, 1} / c_{h}^{T, 2+3} \rightarrow \pm \infty$.

\subsubsection{Evaluation of the $c_{h}^{T}$ contribution}

All following distributions are plotted over the $E c$ number. Evaluated are the non-adiabatic contribution $c_{h}^{S R A} / c_{h}$ in figure $4(a)$, the contributions of $c_{h}^{T, 1} / c_{h}$ and $c_{h}^{T, 2+3} / c_{h}$ in panel $(b)$ and its ratio $c_{h}^{T, 1} / c_{h}^{T, 2+3}$ in panel (c); the contribution of $c_{h}^{T, 3}$ will be discussed afterwards. Contrary to figure 2, ZPG cases are plotted in red, APG cases in blue, and the strongly cooled supersonic ZPG case in orange. The red and orange coloured ZPG cases are discussed first, the blue coloured pressure-gradient cases afterward.

ZPG cases. Six ZPG cases are considered at $M_{e}=0.3,0.5,0.95$ and 2.0 with various relative wall temperatures of $\left(\bar{T}_{r}-T_{w}\right)=+2 \mathrm{~K},+10 \mathrm{~K},+20 \mathrm{~K}$ and $-308 \mathrm{~K}$. As previewed 


\section{Momentum and energy transfer in compressible TBLs}
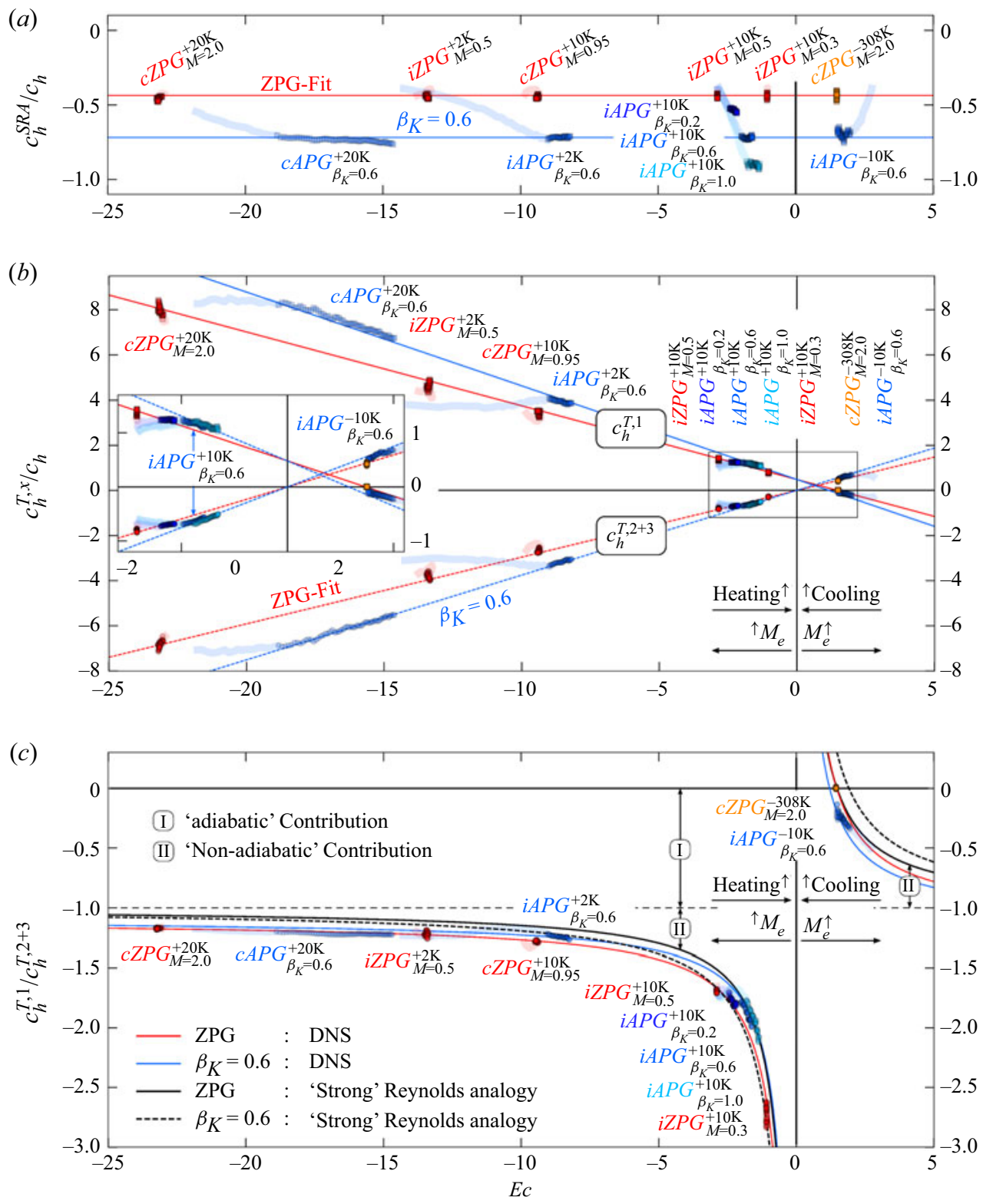

Figure 4. Evaluation of the turbulent contribution $c_{h}^{T}$ according to (6.1) as a function of the Eckert number $E c=(\gamma-1) M_{e}^{2} T_{e} /\left(\bar{T}_{r}-T_{w}\right)$. Depicted are the $c_{h}^{S R A} / c_{h}$ term according to (6.5) in panel (a), the portions $c_{h}^{T, 1} / c_{h}$ and $c_{h}^{T, 2+3} / c_{h}$ in panel (b) and their ratio $c_{h}^{T, 1} / c_{h}^{T, 2+3}$ in panel (c). Symbols represent results evaluated at every 20th numerical grid point in the streamwise direction in regions of self-similarity. ZPG cases are coloured red, APG cases blue and the strongly cooled supersonic case yellow. Blue and red lines are linear best fits according to $(6.12 a, b)-(6.17)$.

in the previous section, the heat-flux modelling term of the SRA $c_{h}^{S R A} / c_{h}$ is independent of $E c$ for all ZPG cases in panel (a). According to (6.8), it is approximated as

$$
\left.\frac{c_{h}^{S R A}}{c_{h}}\right|_{Z P G} \approx-0.44 .
$$




\section{Wenzel, T. Gibis and M. Kloker}

Next, the ratios of the enthalpy fluctuations $c_{h}^{T, 1} / c_{h}$ and the kinetic-energy fluctuations $c_{h}^{T, 2+3} / c_{h}$ depicted in panel $(b)$ are discussed. As predicted in $\S 6.1 .1$, these two terms show opposite behaviour and scale in good approximation linearly with the $E c$ number for all cases. The trends are approximated by the red lines according to (6.7) and (6.9) with

$$
\left.\frac{c_{h}^{T, 1}}{c_{h}}\right|_{Z P G} \approx-0.32696 E c+0.48 \text { and }\left.\frac{c_{h}^{T, 2+3}}{c_{h}}\right|_{Z P G} \approx 0.29565 E c .
$$

While the slope of $c_{h}^{T, 2+3} / c_{h}$ is positive, the slope of $c_{h}^{T, 1} / c_{h}$ is negative and approximately $10 \%$ steeper. The latter differs from the SRA, which predicts an equal absolute slope of $c_{h}^{T, 1} / c_{h}$ and $c_{h}^{T, 2+3} / c_{h}$ (limit of the SRA for $c_{h}^{T, 1} / c_{h}^{T, 2(+3)}(E c \rightarrow \pm \infty)=-1$ ). The good agreement of all data shown with the linear approximations confirms the functional forms introduced, as well as the definition of the $E c$ number introduced. Of particular importance is the behaviour of $(6.12 a, b)$ for small absolute $E c$ numbers. For $E c$ tending towards $\pm 0\left(E c=0\right.$ is only possible if $\left.M_{e}=0\right)$, the turbulent contribution of the kinetic energy $c_{h}^{T, 2+3}$ becomes increasingly dominated by the turbulent heat transfer $c_{h}^{T, 1}$, which would contribute $48 \%$ to the overall $c_{h}$ for a hypothetical $E c$ number of 0 . For the ZPG cases, the non-zero contribution of $c_{h}^{T, 1}$ at $E c=0$ approximately equalizes the constant $c_{h}^{S R A} / c_{h} \approx-0.44$. This suggests that the non-zero enthalpy fluctuations $c_{h}^{T, 1}$ at $E c \rightarrow \pm 0$ are adequately represented by the non-adiabatic contribution $c_{h}^{S R A}$ in the SRA; see (6.9).

Next, the $c_{h}^{T, 1} / c_{h}^{T, 2+3}$ ratio depicted in figure $4(c)$ is discussed. Using the approximations for $c_{h}^{T, 1} / c_{h}$ and $c_{h}^{T, 2+3} / c_{h}$ from $(6.12 a, b)$, the approximation for the DNS data results in

$$
\left.\frac{c_{h}^{T, 1}}{c_{h}^{T, 2+3}}\right|_{Z P G} \approx-1.1059+\frac{1.6235}{E c}
$$

its distribution is depicted as a red solid line in panel $(c)$. Using the approximations for $c_{h}^{S R A} / c_{h}$ and $c_{h}^{T, 2+3} / c_{h}$ in (6.11) and $(6.12 a, b)$, yielding $C_{1}\left(\beta_{K}\right) \approx 0.29565$ and $C_{2}\left(\beta_{K}\right) \approx$ -0.44 , the $c_{h}^{T, 1} / c_{h}^{T, 2+3}$ ratio predicted by the SRA (6.10) results in

$$
\left.\frac{c_{h}^{T, 1}}{c_{h}^{T, 2+3}}\right|_{Z P G} ^{S R A} \approx-1+\frac{1}{E c} \frac{C_{2}\left(\beta_{K}\right)}{C_{1}\left(\beta_{K}\right)}=-1+\frac{1.4882}{E c} ;
$$

its distribution is depicted as a black solid line in panel $(c)$. While there are some differences in the constants between (6.13) and (6.14), the characteristic behaviour of the resulting hyperbolas is the same: for $E c \rightarrow \pm \infty$, the ratio tends towards a constant value; with -1.1059 for the DNS data, this value is approximately $10 \%$ lower than predicted by the SRA. For these cases, both the turbulent heat transfer and the turbulent kinetic-energy transfer are of the same order and act in the opposite wall-normal direction. For $E c \rightarrow \pm 0$, both distributions exhibit a singularity, when the turbulent heat transfer greatly exceeds the turbulent kinetic-energy transfer. The different constants in the numerators of the $E c$-number-dependent terms (1.6235 in (6.13) and 1.4882 in (6.14)) only have a minor influence on the slope of the respective curves. Particular attention should be paid to the $E c$-number range $0<E c \lesssim 1.5$. Due to the offset of $c_{h}^{T, 1}$ at $E c=0$, both terms $c_{h}^{T, 1}$ and $c_{h}^{T, 2+3}$ are positive in the considered range in panel $(b)$, causing also a positive ratio in panel $(c)$. Hence, for cases with $0<E c \lesssim 1.5$, both the turbulent heat transfer and the 
turbulent kinetic-energy transfer act in the same wall-normal direction; see $\S 7.1$ for further discussion.

Pressure-gradient cases. Next, the blue pressure-gradient cases in figure 4 are considered. Depicted are cases with $\beta_{K}=0.2,0.6$ and 1.0. For $\beta_{K}=0.2$ and 1.0 only a single subsonic case is shown. For $\beta_{K}=0.6$ four cases are shown, three subsonic ones with $\left(\bar{T}_{r}-T_{w}\right)=+2 \mathrm{~K},+10 \mathrm{~K}$ and $-10 \mathrm{~K}$, and a supersonic one with $\left(\bar{T}_{r}-T_{w}\right)=+20 \mathrm{~K}$. Implied by their equal $\beta_{K}$ value, the four $\beta_{K}=0.6$ cases are expected to exhibit similar behaviour; they are approximated by a single set of linear fits depicted as blue lines in figure 4. In panel $(a)$, the $c_{h}^{S R A} / c_{h}$ term is approximated as a constant,

$$
\left.\frac{c_{h}^{S R A}}{c_{h}}\right|_{\beta_{K}=0.6} \approx-0.72
$$

which is notably smaller than the value of -0.44 found for the ZPG cases in (6.8). Depicted in panel $(b)$, the approximations for $c_{h}^{T, 1} / c_{h}$ and $c_{h}^{T, 2+3} / c_{h}$ are

$$
\left.\frac{c_{h}^{T, 1}}{c_{h}}\right|_{\beta_{K}=0.6} \approx-1.27 \times 0.32696 E c+0.48 \text { and }\left.\frac{c_{h}^{T, 2+3}}{c_{h}}\right|_{\beta_{K}=0.6} \approx 1.27 \times 0.29565 E c,
$$

yielding the $c_{h}^{T, 1} / c_{h}^{T, 2+3}$ ratio depicted in panel $(c)$,

$$
\left.\frac{c_{h}^{T, 1}}{c_{h}^{T, 2+3}}\right|_{\beta_{K}=0.6} \approx-1.1059+\frac{1.2784}{E c} .
$$

With the approximations for $c_{h}^{S R A} / c_{h}$ and $c_{h}^{T, 2+3} / c_{h}$ in $(6.15)$ and $(6.16 a, b)$, yielding $C_{1}\left(\beta_{K}\right) \approx 1.27 \times 0.29565$ and $C_{2}\left(\beta_{K}\right) \approx-0.72$, the $c_{h}^{T, 1} / c_{h}^{T, 2+3}$ ratio predicted by the SRA (6.10) becomes

$$
\left.\frac{c_{h}^{T, 1}}{c_{h}^{T, 2+3}}\right|_{\beta_{K}=0.6} ^{S R A} \approx-1+\frac{1}{E c} \frac{C_{2}\left(\beta_{K}\right)}{C_{1}\left(\beta_{K}\right)}=-1+\frac{1.9174}{E c}
$$

see the black dashed line in panel $(c)$.

As shown in figure $4(b)$, the slope of the approximations $(6.16 a, b)$ differs distinctly between the pressure-gradient and ZPG cases; for the APG cases with $\beta_{K}=0.6$, both slopes are approximately $27 \%$ steeper than in the ZPG cases; compare $(6.12 a, b)$ and $(6.16 a, b)$. Considering $E c=0$, the zero offset of the $c_{h}^{T, 1} / c_{h}$ contribution $(\approx 0.48)$ is approximately the same for $\beta_{K}=0.6$ as for the ZPG cases. In contrast to the ZPG cases discussed before, this offset is not correctly balanced by the heat-transfer modelling term $c_{h}^{S R A}$ by the SRA in $(6.15)(\approx-0.72)$. Due to the large schematic similarity between the APG and ZPG cases in panel $(b)$, however, the ratio $c_{h}^{T, 1} / c_{h}^{T, 2+3}$ is very similar for the APG and ZPG cases in panel (c); compare the red and blue lines. For $E c \rightarrow \pm \infty$, both the APG and ZPG cases tend to a comparable value of approximately -1 .1. For small $E c$ numbers with $|E c| \lesssim 5$, in contrast, the deviations can be large. Note that pressure-gradient influences on $c_{h}^{T, 1} / c_{h}^{T, 2+3}$ behave qualitatively differently between DNS data and the 


\section{Wenzel, T. Gibis and M. Kloker}

prediction of the SRA. For the DNS data, the influence of APGs moves the vertex of the respective hyperbola closer to the origin compared with the ZPG cases, while the SRA predicts the opposite behaviour; compare the red and blue lines and the black dashed and solid black lines. This behaviour can be partly attributed to the mismatch between the contributions of $c_{h}^{T, 1}$ and $c_{h}^{S R A}$ at $E c=0$, implying that pressure-gradient effects are not adequately incorporated in the present formulation of the SRA chosen; see $\S 7$ for a more detailed discussion. Nevertheless, from an integral point of view, the underlying schematic, predicting the trends depicted in panel $(c)$, can be assessed to apply to both the ZPG and APG cases considered. It is noteworthy that this holds in good approximation for both regions with self-similar flow (symbols) and regions with non-self-similar flow (thick blue lines).

Briefly summarizing the main result for both the ZPG cases and the APG cases, figure 4 provides a schematic to evaluate how the turbulent transfer processes within the total-enthalpy equation are related to each other for a wide parameter range as a function of the local Ec number. In $\S 7$ some further remarks are made on how far the behaviour shown for the integral progressions of $c_{h}^{T, 1} / c_{h}^{T, 2(+3)}$ depicted can be transferred to the local profiles of its integrands $\bar{\rho} \widetilde{v}^{\prime \prime} h^{\prime \prime}$ and $E c \tilde{u} \bar{\rho} u^{\prime \prime} v^{\prime \prime}$.

Sum of $c_{h}^{T}$ and influence of the $c_{h}^{T, 3}$ term. In the previous section, both the triple-fluctuation terms of the mean kinetic energy $c_{h}^{T, 2}$ and the turbulent kinetic energy $c_{h}^{T, 3}$ are considered as a single term $c_{h}^{T, 2+3}$. This differs from the classical approach where the term of the fluctuating turbulent kinetic energy $\left(c_{h}^{T, 3}\right)$ is usually neglected, raising the question of how large the influence of $c_{h}^{T, 3}$ is and on which factors its influence depends.

For the $c_{h}^{T, 2+3}$ distribution depicted in figure $4(b)$, the contribution of $c_{h}^{T, 3}$ is only minor. If only $c_{h}^{T, 2}$ is evaluated instead of $c_{h}^{T, 2+3}$, the slope of the $c_{h}^{T, 2+3} / c_{h}$ approximation in $(6.12 a, b)$ is reduced by approximately $3 \%$. For the sum of all turbulent terms $c_{h}^{T}=c_{h}^{T, 1}+$ $c_{h}^{T, 2}+c_{h}^{T, 3}$, however, which represents the contribution of the total-enthalpy fluctuations in (3.8), $c_{h}^{T, 3}$ can make up a substantial part, since the counteracting contributions of $c_{h}^{T, 1}$ and $c_{h}^{T, 2}$ largely cancel. To illustrate the share of $c_{h}^{T, 3}$ in $c_{h}^{T}$, both the sum $c_{h}^{T} / c_{h}=$ $\left(c_{h}^{T, 1}+c_{h}^{T, 2}+c_{h}^{T, 3}\right) / c_{h}$ (red dotted approximation) and its contribution $c_{h}^{T, 3} / c_{h}$ (red solid approximation) are depicted as functions of the $E c$ number in figure 5. The red ZPG trend of $c_{h}^{T} / c_{h}$ is computed from the sum of its components $(6.12 a, b)$, and the red trend for $c_{h}^{T, 3} / c_{h}$ is approximated by a linear fit:

$$
\frac{c_{h}^{T}}{c_{h}} \approx-0.03131 E c+0.48, \quad \frac{c_{h}^{T, 3}}{c_{h}} \approx-0.01 E c .
$$

As both the $c_{h}^{T} / c_{h}$ and the $c_{h}^{T, 3} / c_{h}$ contributions exhibit an $E c$-number dependence, the percentage share of $c_{h}^{T, 3}$ in the overall $c_{h}^{T}$ depends on the local $E c$ number. At a hypothetical $E c=0, c_{h}^{T, 3}$ makes no contribution to $c_{h}^{T}$. At $E c=-25, c_{h}^{T, 3}$ contributes approximately $20 \%$ to the overall $c_{h}^{T}$ and thus $25 \%$ to the overall $c_{h}$. For the subsonic pressure-gradient cases in figure 5 , both $c_{h}^{T} / c_{h}$ and $c_{h}^{T, 3} / c_{h}$ are almost unaffected by the pressure-gradient strength in regions of self-similar flow; all results are in good agreement with the red approximations for the ZPG cases. The supersonic pressure-gradient case at $E c \approx-17$, in 
Momentum and energy transfer in compressible TBLs

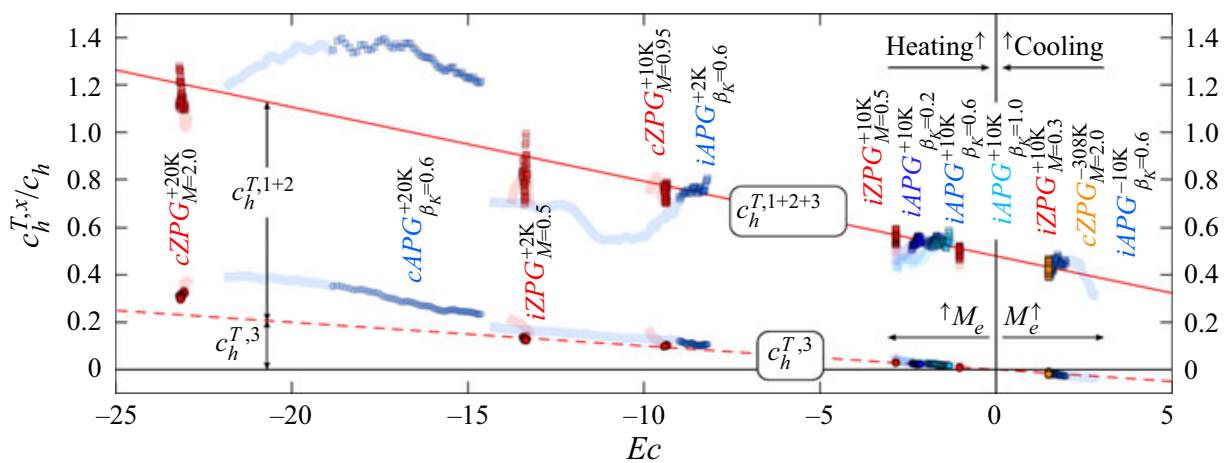

Figure 5. Contribution of $c_{h}^{T, 3}$ to $c_{h}^{T}=c_{h}^{T, 1+2+3}$ according to (6.1) as a function of the Eckert number $E c=(\gamma-1) M_{e}^{2} T_{e} /\left(\bar{T}_{r}-T_{w}\right)$. Symbols represent results evaluated at every 20th numerical grid point in the streamwise direction in regions of self-similarity. ZPG cases are coloured red, APG cases blue and the strongly cooled supersonic case yellow. The red solid line is the sum of the ZPG approximations according to $(6.12 a, b)$, and the dashed red line approximates the portion of the $c_{h}^{T, 3}$ term.

contrast, greatly deviates from the approximation shown. However, it is emphasized that $c_{h}^{T}$ is the sum of two large opposite terms, which makes its distribution very sensitive. Although Mach-number influences have been almost completely eliminated in previous representations by scaling with $c_{f}$ and/or $c_{h}$, they seem to be of relevance for the quantities considered here, at least for the supersonic pressure-gradient case.

\subsection{Evaluation of the overall $c_{h}$ identity}

In the following, the overall integral identity of $c_{h}$ (3.8) is evaluated, putting the turbulent-convection term $c_{h}^{T}$ in its overall context. All quantities evaluated are normalized with $c_{h}$ in figure $6(a)$ and with $1 / 2 c_{f}$ in panel $(b)$.

First, figure $6(a)$ is discussed. Using $(6.19 a, b)$ for $c_{h}^{T} / c_{h}$, the remaining ZPG approximations depicted are fitted by

$$
\frac{c_{h}^{L}}{c_{h}} \approx-0.003913 E c+0.03, \quad \frac{c_{h}^{D}+c_{h}^{M}}{c_{h}} \approx 0.03522 E c+0.49 .
$$

The boundary-layer term $c_{h}^{L}$ is negligibly small for small absolute $E c$ numbers, but increases notably with decreasing $E c$ number; for example, it contributes with approximately $12 \%$ at $E c=-25$, a considerable part of $c_{h}$. Hence, the dominant behaviour occurring with varying $E c$ number is the variation of $c_{h}^{T} / c_{h}$ and $\left(c_{h}^{D}+c_{h}^{M}\right) / c_{h}$. Like $c_{h}^{T} / c_{h}$, both $c_{h}^{L} / c_{h}$ and $\left(c_{h}^{D}+c_{h}^{M}\right) / c_{h}$ are virtually unaffected by the strength of the pressure gradient for the subsonic cases; compare the blue dots and red lines.

With $2 c_{h}^{x} / c_{f}=\left(c_{h}^{x} / c_{h}\right) s$, panel $(b)$ represents a multiplication of the $c_{h}^{x} / c_{h}$ distributions depicted in panel $(a)$ by $s$; the same holds for the approximations $(6.19 a, b)$ and $(6.20 a, b)$. As the sum of all contributions results in the Reynolds analogy factor $s=2 c_{h} / c_{f}$, see Wenzel et al. (2021), the individual portions $2 c_{h}^{x} / c_{f}$ quantify their relative contributions to $s$. While in panel $(a)$ all distributions add up to 100 per cent and thus allow a simple comparison between the different cases, the pressure-gradient and ZPG cases are fanned out in panel $(b)$ due to the strong pressure-gradient dependence of $s$. This results in a separate set of curves for each $\beta_{K}$, adding up to the respective $s$ value; again, only the sets for $\beta_{K}=0$ and $\beta_{K}=0.6$ are approximated by red and blue lines, respectively. Since the 


\section{Wenzel, T. Gibis and M. Kloker}
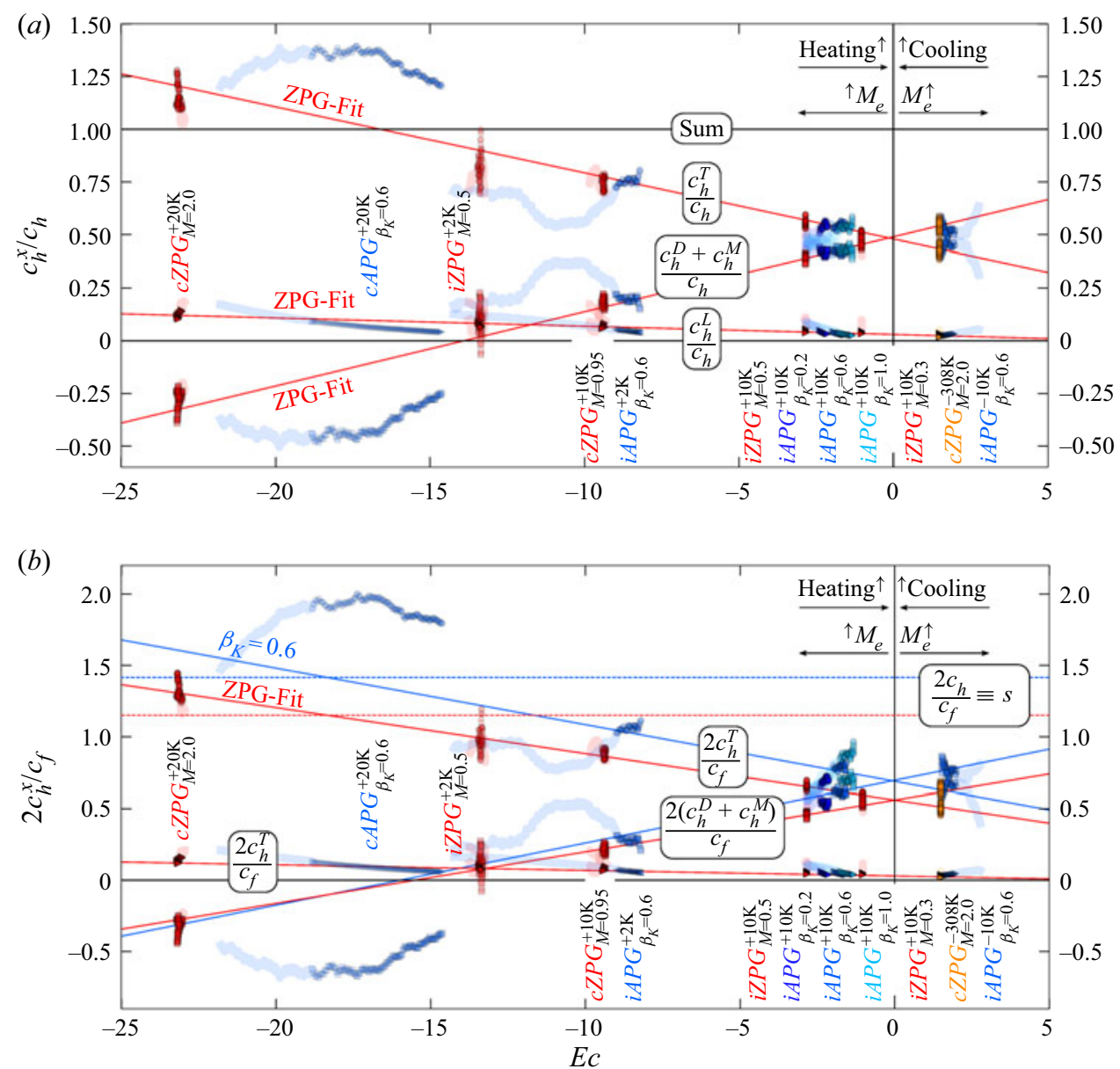

Figure 6. Evaluation of the $c_{h}$ identity according to (3.8) as function of the Eckert number $E c=(\gamma-$ $1) M_{e}^{2} T_{e} /\left(\bar{T}_{r}-T_{w}\right)$. The ratio of $c_{h}^{x} / c_{h}$ is given in panel $(a), 2 c_{h}^{x} / c_{f}$ in panel $(b)$. Symbols represent results evaluated at every 20th grid point in the streamwise direction in regions of self-similarity. ZPG cases are coloured red, APG cases blue and the strongly cooled supersonic case yellow. Red and blue lines linearly approximate ZPG and $\beta_{K}=0.6$ cases.

Reynolds analogy factor is only weakly influenced by the local $E c$ number, the sum of the respective curves is also virtually invariant to the $E c$ number for cases with the same $\beta_{K}$ in near-adiabatic conditions. To what extent the course of the pressure-gradient cases would be affected under strongly non-adiabatic conditions cannot be evaluated based on the available data, however. At least for the strongly cooled supersonic ZPG case, no influence of the wall temperature can be determined.

\section{Discussion}

Having presented the integral results in the previous section, some of the resulting consequences and implications are examined in more detail in the following. In $\S 7.1$, trends obtained from the integral analyses are related to local, wall-normal distributions. In $\S 7.2$, results from the integral $c_{f}$ and $c_{h}$ analysis are related to each other. 


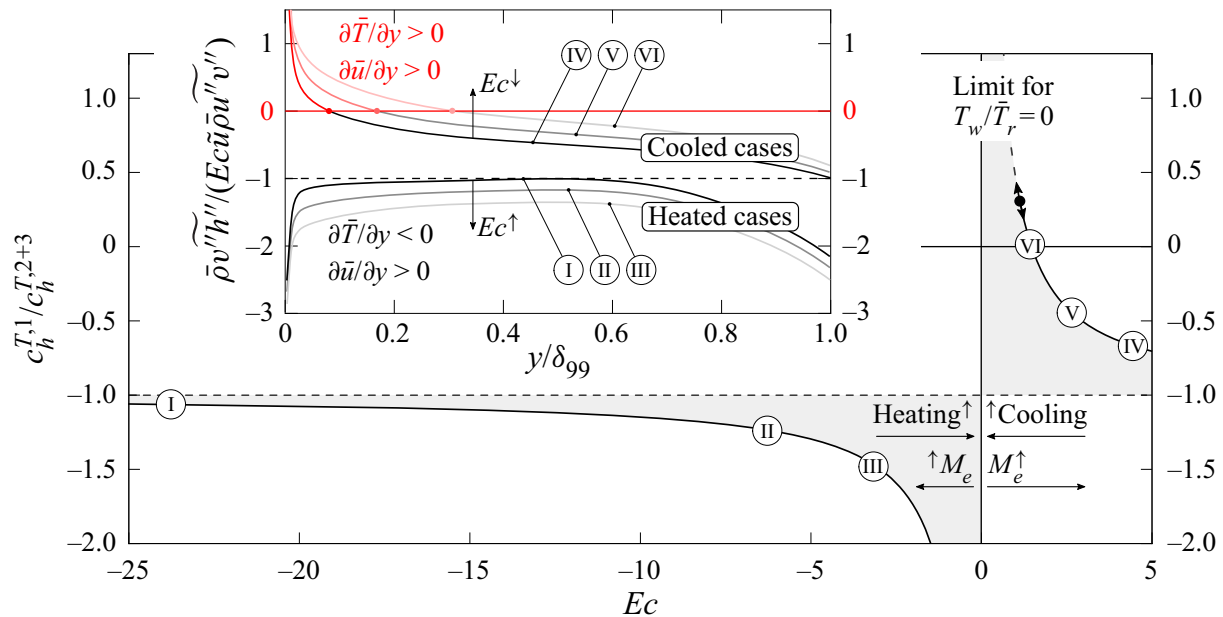

Figure 7. Schematic diagram of figure $4(c)$. Depicted is the integral, $E c$-number-dependent trend of $c_{h}^{T, 1} / c_{h}^{T, 2+3}$ predicted by the SRA (6.6), as well as a schematic plot visualizing the principal behaviour of the ratio of its integrands $\bar{\rho} v^{\prime \prime} h^{\prime \prime} /\left(E c \tilde{u} \bar{\rho} u^{\prime \prime} v^{\prime \prime}\right)$ at six distinct $E c$ numbers (I)-(VI).

\subsection{Integral trends and their local meaning}

All trends found so far are based on the integral point of view, ignoring the particular shape of the wall-normal distribution of the respective integrand. This raises the question of to what degree the wall-normal distributions reflect the trends obtained from the integral perspective. For an assessment, the main characteristics observed in figure $4(c)$ for the integral trends are repeated in figure 7 alongside the wall-normal distributions of the respective integrands in a schematic diagram. Depicted are the $E c$-number-dependent trend of $c_{h}^{T, 1} / c_{h}^{T, 2+3}$ predicted by the SRA and the schematic behaviour of its integrand $\bar{\rho} v^{\prime \prime} h^{\prime \prime} /\left(E c \tilde{u} \bar{\rho} u^{\prime \prime} v^{\prime \prime}\right)$ at six distinct $E c$ numbers.

Following the intuition given by Prandtl's mixing-length model, the signs of $E c \tilde{u} \bar{\rho} u^{\prime \prime} v^{\prime \prime}$ and $\bar{\rho} v^{\prime \prime} h^{\prime \prime}$ depend on the signs of $\partial \bar{u} / \partial y$ and $\partial \bar{T} / \partial y$, respectively, making the interpretation of the different cases straightforward. For heated cases $(E c<0), \partial \bar{T} / \partial y$ and $\partial \bar{u} / \partial y$ are of opposite signs throughout the entire boundary layer, implying a negative sign of $\bar{\rho} v^{\prime \prime} h^{\prime \prime} /\left(E c \tilde{u} \bar{\rho} u^{\prime \prime} v^{\prime \prime}\right)$. For $E c \rightarrow-\infty$ (near adiabatic), the ratio is close to -1 , and for $E c \rightarrow-0$, the ratio is reduced; see cases (I) $\rightarrow$ (III). For cooled cases $(E c>0)$, $\partial \bar{T} / \partial y$ and $\partial \bar{u} / \partial y$ are of the same sign in the near-wall region, also causing regions with a positive sign of $\bar{\rho} v^{\prime \prime} h^{\prime \prime} /\left(E c \tilde{u} \bar{\rho} \widetilde{u}^{\prime \prime} v^{\prime \prime}\right)$ near the wall. For $E c \rightarrow+\infty$ (near adiabatic), the negative regions dominate the positive regions, resulting in a ratio close to -1 , whereas for cases with $E c \rightarrow+0$, the positive regions increasingly dominate the negative regions; see cases $(\mathrm{IV}) \rightarrow(\mathrm{VI})$. From an integral point of view, an $E c$ number of approximately 1.5 represents cases where the wall-proximal positive and the wall-distant negative contributions equalize each other. According to figure 3, this is only (at least theoretically) possible for cases with $M_{e} \lesssim 3.4$, where $E c\left(T_{w} / \bar{T}_{r}=0\right) \lesssim 1.5$. It is mentioned that this switch of the integral terms does not signal an abrupt change in the local behaviour. Note that the topological differences due to the change of the sign of $\partial \bar{T} / \partial y$ between cooled and heated cases are hidden in the integral evaluation. In the integral sense, cooling and heating appear to behave in the same way, and only the sign of the additional energy flux introduced is altered.

To assess to what extent the integral trends found for $c_{h}^{T, 1} / c_{h}^{T, 2+3}$ are transferable to the actual ratio of its integrands $\bar{\rho} v^{\prime \prime} h^{\prime \prime} /\left(E c \tilde{u} \bar{\rho} u^{\prime \prime} v^{\prime \prime}\right)$, the wall-normal distributions 
(a)

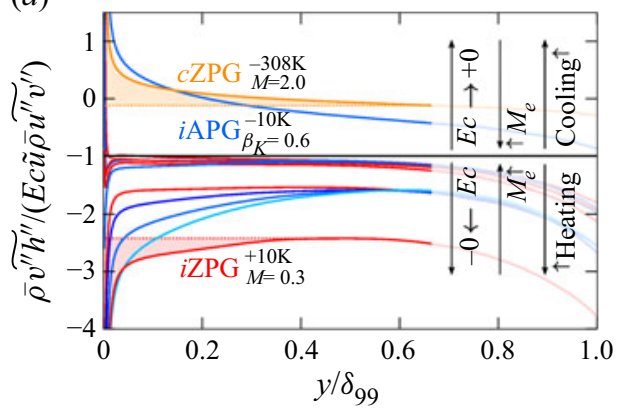

(b)

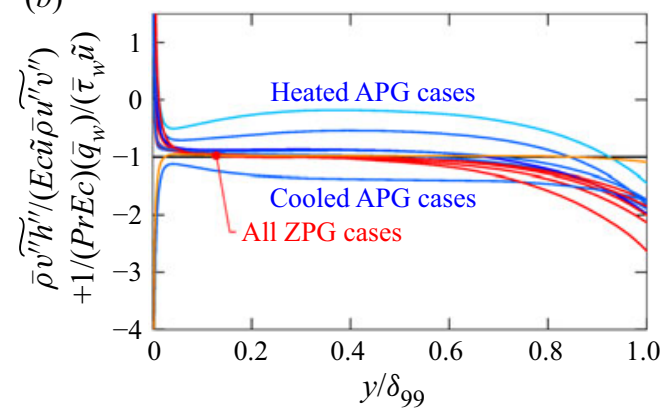

Figure 8. Wall-normal distributions of $\bar{\rho} v^{\prime \prime} h^{\prime \prime} /\left(E c \tilde{u} \bar{\rho} u^{\prime \prime} v^{\prime \prime}\right)$ in panel $(a)$ and $\bar{\rho} v^{\prime \prime} h^{\prime \prime} /\left(E c \tilde{u} \bar{\rho} \widetilde{u}^{\prime \prime} v^{\prime \prime}\right)+$ $\bar{q}_{w} /\left(\operatorname{PrEc} \bar{\tau}_{w} \tilde{u}\right)$ in panel $(b)$; see (7.1). All distributions are averaged in the streamwise direction in regions of self-similarity. ZPG cases are coloured red, APG cases blue and the strongly cooled supersonic case orange. The red and orange shaded regions in panel (a) exemplarily emphasize the deviation from a constant.

of $\bar{\rho} \widetilde{v^{\prime \prime} h^{\prime \prime}} /\left(E c \tilde{u} \bar{\rho} u^{\prime \prime} v^{\prime \prime}\right)$ are depicted in figure $8(a)$. Only regions for $y / \delta_{99} \leq 0.6$ are meaningful, where the denominator is not close to zero. Following the prediction of the SRA, the distributions shown are in line with the reasoning expected. Using the Morkovin relation introduced in $\S 6.1 .1$ and neglecting the triple-fluctuation terms, (6.4) simplifies to

$$
\frac{1}{E c} \frac{\bar{\rho} \widetilde{v}^{\prime \prime} h^{\prime \prime}}{\tilde{u} \bar{\rho} \widetilde{u}^{\prime \prime} v^{\prime \prime}} \approx-1-\frac{1}{\operatorname{Pr} E c} \frac{\bar{q}_{w}}{\bar{\tau}_{w}} \frac{1}{\tilde{u}} .
$$

For cases with high absolute $E c$ numbers, the $E c$-number-dependent term in (7.1) becomes negligible and the resulting distributions stay close to -1 for $y / \delta_{99} \lesssim 0.6$. Hence, for high absolute $E c$ numbers, the integral trends closely resemble the distributions of $\bar{\rho} v^{\prime \prime} h^{\prime \prime} /\left(E c \tilde{u} \bar{\rho} u^{\prime \prime} v^{\prime \prime}\right)$. For cases with low absolute $E c$ numbers, e.g. the $i Z P G_{M=0.3}^{+10 \mathrm{~K}}$ case or the $c Z P G_{M=2.0}^{-308 \mathrm{~K}}$ case, the $E c$-number-dependent term in (7.1) 'rounds' the resulting distribution in the near-wall region for $y / \delta_{99} \lesssim 0.2$ with a wall-distance-dependent portion depending on $1 / \tilde{u}$; see the red and orange shaded regions in panel $(a)$. Hence, for small absolute $E c$ numbers, the integral values only estimate the magnitude of the local profiles.

To evaluate (7.1) for pressure gradients, the sum of $\bar{\rho} v^{\prime \prime} h^{\prime \prime} /\left(E c \tilde{u} \bar{\rho} u^{\prime \prime} v^{\prime \prime}\right)$ and $1 /(\operatorname{PrEc})\left(\bar{q}_{w} / \bar{\tau}_{w}\right)(1 / \tilde{u})$ is depicted in figure $8(b)$. As all ZPG cases resemble a constant trend close to -1 independent of the $E c$ number considered, the Morkovin relation (7.1) can be assumed to incorporate effects of $E c$-number variations accurately. For the pressure-gradient cases depicted in blue, however, the distributions differ distinctly from the expected value of -1 , suggesting that the pressure gradient must be accounted for by an additional term in the wall shear stress in (7.1). In the integral analysis, this portion would increase the value of the constant $C_{1}\left(\beta_{K}\right)$ depicted in figure $4(a)$.

\subsection{About the connection between the integral identities of $c_{f}$ and $c_{h}$}

In the previous section the two integral identities for $c_{f}$ and $c_{h}$ were evaluated almost independently of each other. In this section the definition of the Reynolds analogy factor

$$
s=\frac{2 c_{h}}{c_{f}}=\frac{2\left(c_{h}^{L}+c_{h}^{T}+c_{h}^{M}+c_{h}^{D}\right)}{c_{f}^{L}+c_{f}^{T}+c_{f}^{M}+c_{f}^{D}}
$$




\section{Momentum and energy transfer in compressible TBLs}

is utilized to link the two identities. In this way, additional insight is gained regarding the weighting of the respective terms of the momentum and total-enthalpy equations to each other from an integral perspective.

\subsubsection{About the influence of Ec and $\beta_{K}$ on $c_{f}, c_{h}$ and $s$}

As previously discussed and shown in Wenzel et al. (2021), the value of $s=2 c_{h} / c_{f}$ strongly depends on the local pressure-gradient strength and only weakly on the local $E c$ number. Altering the $E c$ number thus alters $c_{h}$ and $c_{f}$ in a proportional manner, leaving $s$ nearly unaffected. An alteration of the local pressure-gradient strength, conversely, alters $c_{h}$ and $c_{f}$ in a non-proportional manner, so that $s$ varies as a function of the local pressure-gradient strength. As shown in this study, the percentage share of $c_{f}^{x}$ in the total $c_{f}$ primarily depends on the pressure gradient and only weakly on the $E c$ number. The percentage share of $c_{h}^{x}$ in the total $c_{h}$, conversely, primarily depends on the $E c$ number and only weakly on the pressure-gradient strength. As a consequence, the following three scenarios can be distinguished for the cases considered here.

(i) Variation of the $E c$ number: $c_{h}$ and $c_{f}$ are influenced in a proportional manner. The percentage shares of $c_{f}^{x}$ in the total $c_{f}$ stay almost constant. The percentage shares of $c_{h}^{x}$ in the total $c_{h}$ scale differently with the $E c$ number, and thus change their relative weight to each other.

(ii) Variation of the pressure gradient: $c_{h}$ and $c_{f}$ are influenced in a non-proportional manner. The percentage shares of $c_{f}^{x}$ in the total $c_{f}$ scale differently with the pressure gradient, and thus change their relative weight to each other. The percentage shares of $c_{h}^{x}$ in the total $c_{h}$ stay almost constant.

(iii) Variation of both $E c$ number and pressure gradient: $c_{h}$ and $c_{f}$ vary by both a proportional contribution due to the pressure gradient and a non-proportional contribution due to the $E c$-number variation. The percentage shares of $c_{f}^{x}$ in the total $c_{f}$ scale differently with the pressure gradient and thus change their relative weight to each other. The percentage shares of $c_{h}^{x}$ in the total $c_{h}$ scale differently with the $E c$ number and thus also change their relative weight to each other.

\subsubsection{About the relation between $c_{h}^{T}$ and $c_{f}^{T}$}

As introduced in $\S 1$, it is the main assumption of the SRA that the total-enthalpy fluctuations $h_{0}^{\prime \prime}$ and the velocity fluctuations $u^{\prime \prime}$ can be directly coupled by the 'strong' assumption of a direct proportionality between $h_{0}^{\prime \prime}$ and $u^{\prime \prime}, k_{1} \approx h_{0}^{\prime \prime} /\left(E c u^{\prime \prime}\right)$. Multiplication by $\bar{\rho} v^{\prime \prime}$ and time averaging yields

$$
k_{1}=\frac{1}{E c} \frac{\bar{\rho} h_{0}^{\prime \prime} v^{\prime \prime}}{\bar{\rho} \widetilde{u^{\prime \prime} v^{\prime \prime}}},
$$

or written in terms of the integral identities,

$$
k_{1}=\frac{1}{E c} \frac{-\frac{2}{y_{b}} \int_{0}^{y_{b}}\left[\bar{\rho} h_{0}^{\prime \prime} v^{\prime \prime}\right] \mathrm{d} y}{-\frac{2}{y_{b}} \int_{0}^{y_{b}}\left[\bar{\rho} \widetilde{\left.u^{\prime \prime} v^{\prime \prime}\right] \mathrm{d} y}\right.}=\frac{1}{E c} \frac{2 c_{h}^{T}}{c_{f}^{T}},
$$

if $k_{1}$ is assumed to be a constant for all wall-normal positions within the boundary layer. Hence, by evaluating the ratio of the turbulent-convection terms of the $c_{h}$ and the $c_{f}$ 

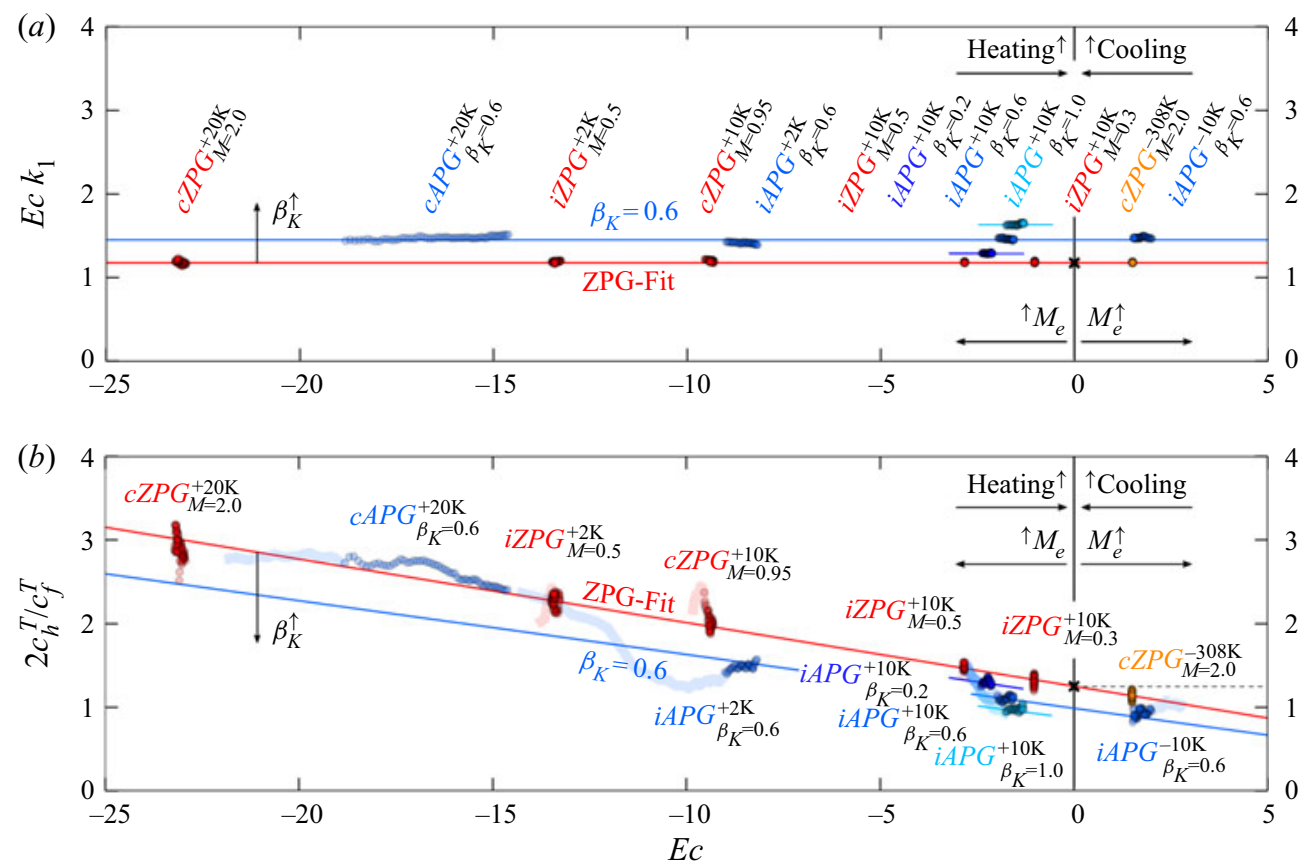

Figure 9. Distribution of $E c k_{1}=-1 / \operatorname{Pr}\left(\bar{q}_{w} / \bar{\tau}_{w}\right)$ in panel (a) and ratio of the turbulent contributions $2 c_{h}^{T}$ and $c_{f}^{T}$ in panel $(b)$, both plotted as a function of the Eckert number $E c=(\gamma-1) M_{e}^{2} T_{e} /\left(\bar{T}_{r}-T_{w}\right)$. Symbols represent results evaluated at every 20th numerical grid point in the streamwise direction in regions of self-similarity. ZPG cases are coloured red, APG cases blue and the strongly cooled supersonic case yellow. Red and blue lines are linear best fits of the ZPG and $\beta_{K}=0.6$ cases, in $(b)$ fitted to the data and in $(a)$ according to (7.5).

identities, $(1 / E c)\left(2 c_{h}^{T} / c_{f}^{T}\right)$, a nominal value for the proportionality constant $k_{1}$ can be obtained. The value $(1 / E c)\left(2 c_{h}^{T} / c_{f}^{T}\right)$ thus represents the value that the proportionality constant of the strong SRA $k_{1}$ would have to take, to satisfy the relation of turbulent correlations in the integral sense; see (7.4). Hence, by comparison of this nominal, integral value for $k_{1}$ and the directly computed value for $k_{1}=-1 /(\operatorname{Pr} E c)\left(\bar{q}_{w} / \bar{\tau}_{w}\right)$ proposed by the SRA, the uncertainty of the 'strong' assumption $k_{1} \approx h_{0}^{\prime \prime} /\left(E c u^{\prime \prime}\right)$ can be assessed from an integral perspective in the considered $E c$-number range. As both $k_{1}$ in (7.3) and $(1 / E c)\left(2 c_{h}^{T} / c_{f}^{T}\right)$ depend on $1 / E c$, both distributions are hyperbolas with the same qualitative behaviour as depicted in figure 7. To allow for a better analysis of their respective shapes, both distributions are plotted multiplied by $E c$ in figure 9 , the directly computed values for $E c k_{1}$ in panel ( $a$ ) and the integral ratio of $2 c_{h}^{T} / c_{f}^{T}$ in panel $(b)$. Using the approximations obtained from the DNS data $(5.3 a-c)$ and $(6.19 a, b)$, the distribution of $2 c_{h}^{T} / c_{f}^{T}$ is approximated by

$$
\frac{2 c_{h}^{T}}{c_{f}^{T}}=\frac{-0.0944 E c+1.408}{s}
$$

in panel $(b)$.

For the directly computed values of $E_{c} k_{1}$ in panel (a), all distributions are constant; for ZPG cases, this value is around 1.18, for APG cases, it is increased. This is in line with 


\section{Momentum and energy transfer in compressible TBLs}

the discussion of figure 4(a). In panel $(b)$, the trends for the ZPG and APG cases both exhibit a clear $E c$-number dependence, increasing for negative $E c$ numbers and decreasing for positive $E c$ numbers. As the $c_{f}^{T}$ distributions in the denominator have been found to be almost $E c$-number independent in figure 2, this trend essentially resembles the qualitative trends found for the $c_{h}^{T}$ distributions in figure 5. For ZPG cases at $E c=0$, the value is comparable to panel (a) (black cross); however, for APG cases, it is reduced in contrast to panel $(a)$. This essentially resembles the same systematic error made by the SRA already seen from the discussion of figures $4(c)$ and $8(b)$.

Based on the curve progressions of $E c k_{1}$ and $2 c_{h}^{T} / c_{f}^{T}$ shown, the progressions of the hyperbolas of $k_{1}$ and $(1 / E c)\left(2 c_{h}^{T} / c_{f}^{T}\right)$ are discussed; only ZPG cases are considered in the following. For the progression of $k_{1}$, panel $(a)$ implies a hyperbola of the form $k_{1} \approx 1.18 / E c$, and thus a hyperbola tending towards \pm 0 in its limit for $E c \rightarrow \pm \infty$. This limit is the direct consequence of the assumption by the SRA that total-enthalpy fluctuations (or in our case $\bar{\rho} h_{0}^{\prime \prime} v^{\prime \prime}$ ) become zero for near-adiabatic cases. For the progression of $(1 / E c)\left(2 c_{h}^{T} / c_{f}^{T}\right)$, panel $(b)$ implies a hyperbola of the form $(1 / E c)\left(2 c_{h}^{T} / c_{f}^{T}\right)=1.408 /(E c s)-0.0944 / s$. This hyperbola has a comparable shape to that of $k_{1}$, but is slightly shifted downwards by an offset of $-0.0944 / s$ over the entire $E c$-number range compared with the hyperbola of $k_{1}$. This $E c$-number-independent offset ultimately quantifies the total-enthalpy fluctuations integrally accounted for in $c_{h}^{T}$, which are non-zero for near-adiabatic cases. Thus, this offset can be understood as a quantification of the integral error made by the SRA through its assumption of vanishing total-enthalpy fluctuations for adiabatic conditions. Whereas this modelling error becomes dominant for large absolute $E c$ numbers for near-adiabatic cases, its influence decreases successively for smaller $E c$ numbers and thus cases with strong heating or cooling.

\section{Conclusions}

To gain deeper insight into the influences of Mach number, heat transfer and pressure gradient on the compressible momentum and total-enthalpy equations, an integral study has been conducted. To this end, newly formulated integral identities for $c_{f}$ and $c_{h}$ have been derived and evaluated based on the dimensionless compressible momentum and total-enthalpy equations, which can be linked by the Reynolds analogy factor $s$. Both identities use the same mathematical approach as the known FIK identity, see Fukagata et al. (2002), but with a twofold repeated integration instead of a threefold one, making its interpretation physically more sound. From a physical point of view, the first integration essentially gives a force/energy balance between the wall and all locations within the integration domain; the second integration represents its average in the wall-normal direction. For both identities, three contributions are distinguished: a turbulent one; a viscous or conductive one; and the one of the sum of the mean convection and spatial development. As the individual terms of the resulting identities stay formally close to the terms of the governing equations for momentum and total enthalpy, the integral identities directly reflect the behaviour of both equations' terms.

Characterizing the behaviour of the momentum equation, the $c_{f}$ identity essentially condenses the findings already discussed in previous studies in a compact manner; see e.g. Wenzel et al. (2019). With increasing APG strength the turbulent portion of $c_{f}$ increases and the mean-convective and spatial-development portions decrease. Furthermore, no 


\section{Wenzel, T. Gibis and M. Kloker}

significant dependence on the Mach number or wall temperature is observed (at least for the near-adiabatic cases considered here) if the $c_{f}$ identity is normalized by $c_{f}$, which essentially corresponds to a normalization with wall quantities and hence Morkovin's transformation for the turbulent terms. As a result, all portions of the $c_{f}$ identity representing the portions of the relevant transfer mechanisms in the momentum equation are (almost) only a function of the pressure-gradient parameter $\beta_{K}=\left(\delta_{K}^{*} / \bar{\tau}_{w}\right)\left(\mathrm{d} p_{e} / \mathrm{d} x\right)$.

Characterizing the behaviour of the total-enthalpy equation, the interpretation of the $c_{h}$ identity is more complex: first, as it is very sensitive and requires the consideration of small, often neglected terms; and second, as the shares of the $c_{h}$ identity are affected by the local Mach number, heat transfer and, in some part, pressure gradients if normalized by $c_{h}$. It is a main conclusion of this study that the particular definition of the Eckert number $E c=u_{e}^{2} /\left(c_{p}\left(\bar{T}_{r}-T_{w}\right)\right)=(\gamma-1) M_{e}^{2} T_{e} /\left(\bar{T}_{r}-T_{w}\right)$ allows us to account for the effects of both Mach number and wall temperature on the boundary layer for a wide parameter range. This essentially implies that flow cases with the same $E c$ number exhibit the same integral behaviour and are thus physically comparable to each other, regardless of how this $E c$ number is achieved by adjusting the Mach number or the wall temperature. For hypersonic Mach numbers, mostly the wall-to-recovery temperature determines the $E_{c}$ number.

Implied by the data considered, all shares of the $c_{h}$ identity (and hence the total-enthalpy equation) show a linear $E c$-number dependence, which can be considered a direct consequence of both the SRA and the Morkovin hypotheses; pressure-gradient effects are small. Applying arguments of the SRA to the individual terms of the $c_{h}$ identity, comprehensive predictions can be made about the integral behaviour of the turbulence terms as a function of the local $E c$ number for a fixed $\beta_{K}$. Conversely, this allows us to estimate the accuracy of the approximation of the SRA to the integral behaviour of the boundary layer for a wide parameter range consisting of Mach number, wall heat transfer and pressure gradients. The main conclusions from the $c_{h}$ identity are the following. (i) Plotted over the $E c$ number, the integral energy transfer caused by the turbulence terms is reflected in an easy-to-understand manner. For adiabatic conditions $(E c \rightarrow \mp \infty)$, the turbulent contributions of heat and kinetic-energy transfer are of the same order and act in the opposite wall-normal direction. For strongly heated or cooled cases $(E c \rightarrow \mp 0)$, the ratio tends to $\mp \infty$, implying that the turbulent contribution of heat transfer tends to largely exceed that of the kinetic-energy transfer. (ii) This behaviour is qualitatively well predicted by the SRA for ZPG cases, but only if the often neglected heat-flux term incorporating non-adiabatic wall conditions is taken into account. Quantitatively, the constants found by the SRA deviate slightly from the DNS data. (iii) Due to the counteracting nature of the turbulent contributions of heat and kinetic-energy transfer for adiabatic cases, the triple-correlation term is non-negligible for the complete turbulence term; its significance increases with increasing absolute $E c$ number. (iv) Up to moderate supersonic Mach numbers, the $E c$ number and thus the relative portions of the terms of the total-enthalpy equation are influenced by both the wall-to-recovery temperature ratio $T_{w} / \bar{T}_{r}$ and the local Mach number. For hypersonic Mach numbers, the influence of the Mach number becomes negligible. (v) For pressure-gradient cases, the qualitative trends are not appropriately predicted by the SRA, implying that a modification for pressure-gradient influences needs to be constructed.

In summary, the integral analysis conducted allows us to represent the relevant transfer mechanisms in a simplified and compact form, clearly visualizing superordinate mechanisms and making them intuitively tangible. Besides providing hints for improved 
turbulence modelling, it forms a schematic that allows us to easily determine the relative importance of the terms of the governing equations, if the local Eckert number $E c=$ $(\gamma-1) M_{e}^{2} T_{e} /\left(\bar{T}_{r}-T_{w}\right)$ and the pressure-gradient parameter $\beta_{K}=\left(\delta_{K}^{*} / \bar{\tau}_{w}\right)\left(\mathrm{d} p_{e} / \mathrm{d} x\right)$ are known.

Acknowledgements. The provision of computational resources on Cray XC40 and NEC SX-Aurora TSUBASA by the Federal High Performance Computing Centre Stuttgart (HLRS) under grant GCS Lamt (LAMTUR), ID 44026, is gratefully acknowledged.

Funding. This work was supported by the Deutsche Forschungsgemeinschaft (grant numbers RI680/38-1, WE6803/1-1).

Declaration of interests. The authors report no conflict of interest.

\section{Author ORCIDs.}

(1) Christoph Wenzel https://orcid.org/0000-0002-2526-952X;

(1) Tobias Gibis https://orcid.org/0000-0001-9281-4279;

Markus Kloker https://orcid.org/0000-0002-5352-7442.

\section{Appendix A. Derivation of the compressible integral identities for $c_{f}$ and $c_{h}$}

\section{A.1. The compressible integral identity for $c_{f}$}

The derivation of the $c_{f}$ identity is based on an $n$-fold repeated integration of (3.5) from 0 to $y_{b}$ for $n>1$ integrations, where $y_{b}$ is the arbitrarily selectable upper integration bound; see (3.3). With the boundary conditions at the wall $\left.\bar{\rho} u^{\prime \prime} v^{\prime \prime}\right|_{y=0}=0$, $1 /\left.\operatorname{Re} \overline{\mu^{\prime}\left(\partial u^{\prime} / \partial y+\partial v^{\prime} / \partial x\right)}\right|_{y=0}=0,1 / \operatorname{Re} \bar{\mu} \partial \bar{v} /\left.\partial x\right|_{y=0}=0$ (assuming that $v$ at the wall is a constant in the streamwise direction) and

$$
-\int_{0}^{y_{b}} \ldots \int_{0}^{y_{n-1}}\left[\left.\frac{1}{R e} \bar{\mu} \frac{\partial \bar{u}}{\partial y}\right|_{y=0}\right] \mathrm{d} y_{n-1} \ldots \mathrm{d} y=-\frac{1}{2} \frac{y_{b}^{n-1}}{(n-1) !} c_{f},
$$

the final $c_{f}$ identity can be expressed as

$$
\begin{aligned}
& c_{f}=\underbrace{2 \frac{n-1}{y_{b}^{n-1}} \frac{1}{\operatorname{Re}} \int_{0}^{y_{b}}\left(y_{b}-y\right)^{n-2}\left[\bar{\mu}\left(\frac{\partial \bar{u}}{\partial y}+\frac{\partial \bar{v}}{\partial x}\right)\right] \mathrm{d} y}_{c_{f}^{L}} \\
& \text { boundary-layer term } \\
& +\underbrace{2 \frac{n-1}{y_{b}^{n-1} \frac{1}{R e}} \int_{0}^{y_{b}}\left(y_{b}-y\right)^{n-2}\left[\overline{\left.\mu^{\prime}\left(\frac{\partial u^{\prime}}{\partial y}+\frac{\partial v^{\prime}}{\partial x}\right)\right]} \mathrm{d} y\right.}_{c_{f}^{V F}}
\end{aligned}
$$




\section{Wenzel, T. Gibis and M. Kloker}

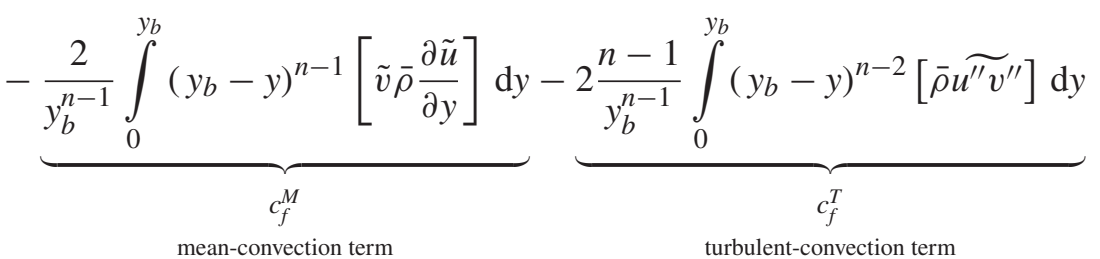

$$
\begin{aligned}
& +\underbrace{\frac{2}{y_{b}^{n-1}} \int_{0}^{y_{b}}\left(y_{b}-y\right)^{n-1}\left[-\tilde{u} \bar{\rho} \frac{\partial \tilde{u}}{\partial x}-\frac{\partial \bar{\rho} \tilde{u}^{\prime \prime} u^{\prime \prime}}{\partial x}+\frac{1}{\operatorname{Re}_{\delta_{99}}} \frac{\partial \bar{\tau}_{x x}}{\partial x}-\frac{1}{\gamma M_{e}^{2}} \frac{\partial \bar{p}}{\partial x}\right] \mathrm{d} y}_{c_{f}^{D}} .
\end{aligned}
$$

\section{A.2. The compressible integral identity for $c_{h}$}

The derivation of the $c_{h}$ identity is based on an $n$-fold repeated integration of (3.6) from 0 to $y_{b}$ for $n>1$ integrations; see (3.3). With the boundary conditions at the wall $\left[\bar{\rho} \widetilde{v}^{\prime \prime} h^{\prime \prime}\right]_{y=0}=0, E c / \operatorname{Re}\left[\bar{\tau}_{x y} \bar{u}+\bar{\tau}_{y y} \bar{v}\right]_{y=0}=0, E c / \operatorname{Re}\left[\overline{\tau_{x y}^{\prime} u^{\prime}}+\overline{\tau_{y y}^{\prime} v^{\prime}}+\overline{\tau_{z y}^{\prime} w^{\prime}}\right]_{y=0}=0$, $E c / 2\left[\bar{\rho} \widetilde{u^{\prime 2} v^{\prime \prime}}+\bar{\rho} \widetilde{v^{\prime \prime}}+\widetilde{\rho} \widetilde{w^{\prime 2} v^{\prime \prime}}\right]_{y=0}=0, \quad E c\left[\tilde{u} \bar{\rho} \widetilde{u^{\prime \prime} v^{\prime \prime}}+\tilde{v} \bar{\rho} \widetilde{v^{\prime \prime 2}}\right]_{y=0}=0$, the neglect of heat-flux fluctuations $q^{\prime}, c_{h}=1 /(\operatorname{RePr}) \bar{\mu} \partial \bar{T} /\left.\partial y\right|_{y=0}$ and

$$
-\left.\int_{0}^{y_{b}} \ldots \int_{0}^{y_{n-1}} \frac{1}{\operatorname{RePr}} \bar{\mu} \frac{\partial \bar{T}}{\partial y}\right|_{y=0} d y_{n-1} \ldots \mathrm{d} y=-\frac{y_{b}^{n-1}}{(n-1) !} c_{h},
$$

the final $c_{h}$ identity becomes

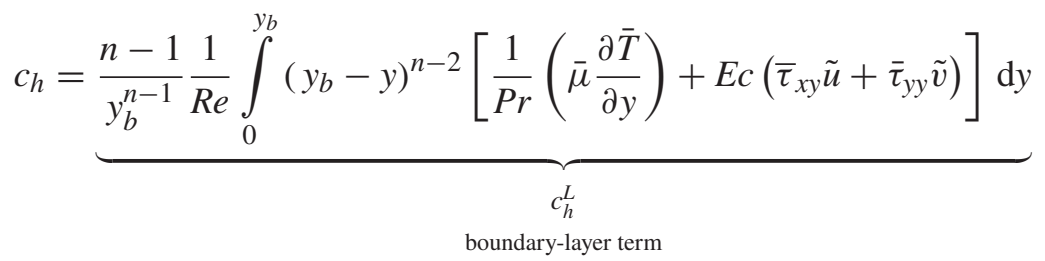

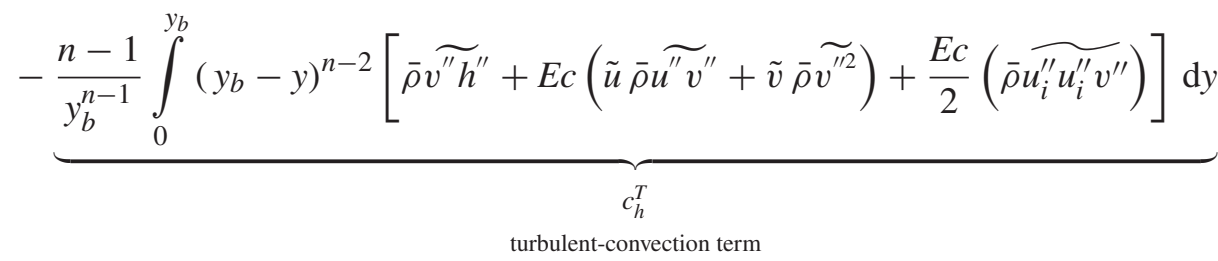

$$
\begin{aligned}
& +\underbrace{\frac{n-1}{y_{b}^{n-1}} \frac{E c}{R e} \int_{0}^{y_{b}}\left(y_{b}-y\right)^{n-2}\left[\overline{\tau_{x y}^{\prime} u^{\prime}}+\overline{\tau_{y y}^{\prime} v^{\prime}}+\overline{\tau_{z y}^{\prime} w^{\prime}}\right] \mathrm{d} y}_{c_{h}^{V F}}
\end{aligned}
$$




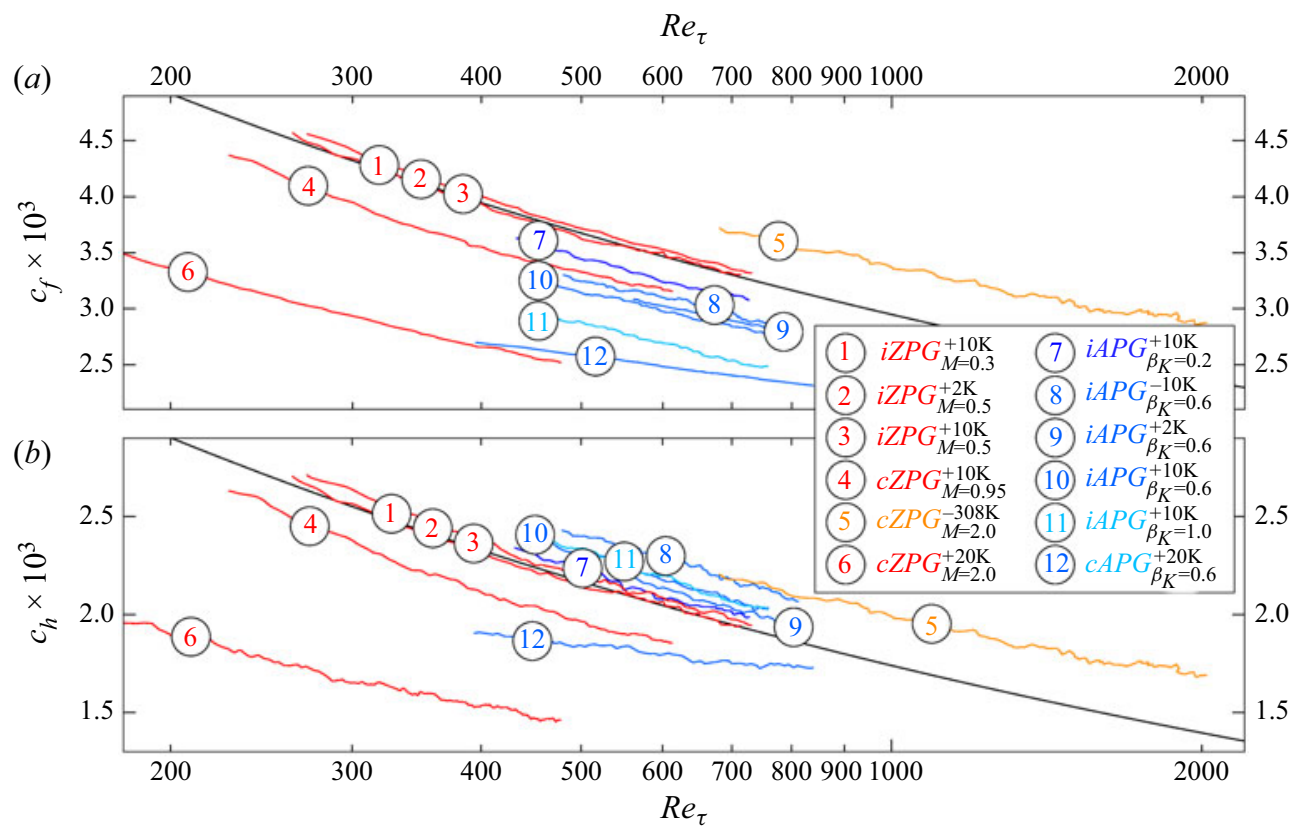

Figure 10. Streamwise evolution of $c_{f}$ and $c_{h}$ in panels $(a, b)$, respectively. A best fit for the subsonic $i Z P G_{M=0.5}^{+10 \mathrm{~K}}$ case is depicted according to $R e_{\tau}=26.515 R e_{\tau}^{-0.318}$ as a black solid line in panel $(a)$, and according to $c_{h}=s\left(c_{f} / 2\right)$ with $s=1.18$ in panel $(b)$; see Wenzel et al. (2021).

$$
\begin{aligned}
& -\underbrace{\frac{1}{y_{b}^{n-1}} \int_{0}^{y_{b}}\left(y_{b}-y\right)^{n-1}\left[\bar{\rho} \tilde{v} \frac{\partial \tilde{h}_{0}}{\partial y}\right] \mathrm{d} y}_{c_{h}^{M}} \\
& \text { mean-convection term }
\end{aligned}
$$

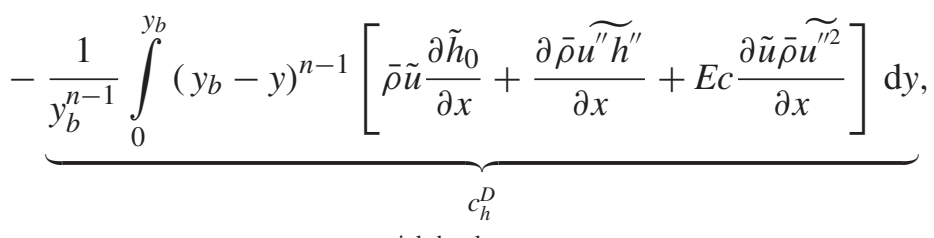

$$
\begin{aligned}
& \text { spatial-development term }
\end{aligned}
$$

where $\bar{\rho} \widetilde{u_{i}^{\prime \prime} u_{i}^{\prime \prime} v^{\prime \prime}}=\bar{\rho} \widetilde{u^{\prime \prime 2} v^{\prime \prime}}+\bar{\rho} \widetilde{v^{\prime \prime 3}}+\widetilde{\rho} \widetilde{w^{\prime \prime 2} v^{\prime \prime}}$.

\section{Appendix B. Additional plots}

\section{B.1. Distributions of $c_{f}$ and $c_{h}$}

In figure 10, the streamwise evolutions of the $c_{f}$ and $c_{h}$ distributions are depicted versus $R e_{\tau}$. For visual comparison, a best fit of the subsonic $i Z P G_{M=0.5}^{+10 \mathrm{~K}}$ case is depicted according to $R e_{\tau}=26.515 R e_{\tau}^{-0.318}$ as a black solid line in panel $(a)$, and according to $c_{h}=s\left(c_{f} / 2\right)$ with $s=1.18$ in panel $(b)$; see Wenzel et al. (2021). A detailed discussion of both the $c_{f}$ and $c_{h}$ distributions is given in Wenzel et al. (2019) and Wenzel et al. (2021), respectively. 


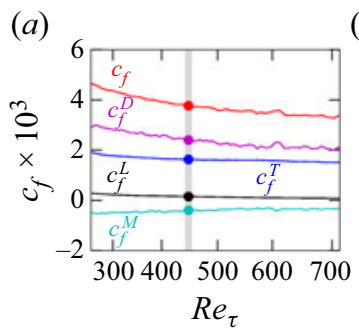

(b)

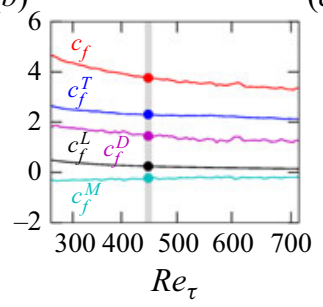

(c)

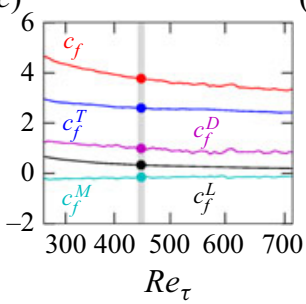

(d)

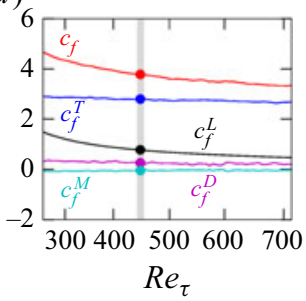

Figure 11. Evaluation of the $c_{f}$ identity (A2) for the subsonic $i Z P G_{M=0.5}^{+10 K}$ case for (a) twofold, (b) threefold, (c) fourfold and $(d)$ tenfold repeated integrations. Values at $R e_{\tau}=450$ are marked for a visual comparison.

(a)

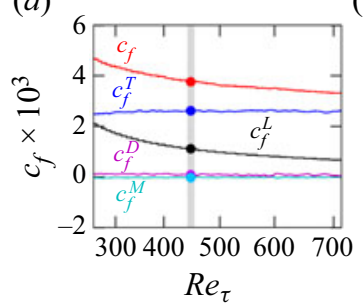

(b)

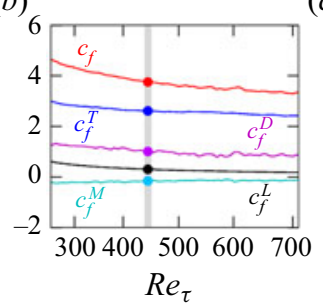

(c)

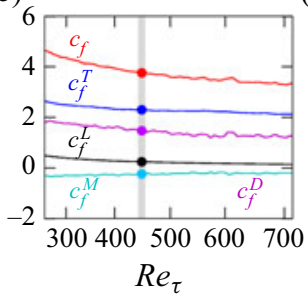

(d)

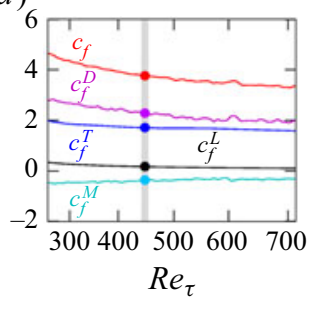

Figure 12. Evaluation of the $c_{f}$ identity (3.8) for the subsonic $i Z P G_{M=0.5}^{+10 \mathrm{~K}}$ case, integrated up to $(a) y_{b}=$ $0.2 \delta_{99},(b) y_{b}=1.0 \delta_{99},(c) y_{b}=1.3 \delta_{99}$ and $(d) y_{b}=2.0 \delta_{99}$. Values at $R e_{\tau}=450$ are marked for a visual comparison.

\section{B.2. Remarks on the number of repeated integrations}

Exemplarily evaluated for $n=2,3,4$ and 10 for the subsonic $i Z P G_{M=0.5}^{+10 \mathrm{~K}}$ case, the streamwise evolution of the integral identity (A2) is evaluated in figure 11 for $c_{f}$ as a function of $R e_{\tau}$. According to Cauchy's formula for repeated integration in (3.3), variations in $n$ essentially only affect the exponent of the weighting terms $\left(y_{b}-y\right)^{n-1}$. Thus, the greater the number of repeated integrations is, the more the near-wall area of the individual terms is weighted. Consequently, the proportions of the $c_{f}^{T}$ and the $c_{f}^{L}$ terms become increasingly dominant for larger $n$, both of which have their maximum in the near-wall region.

\section{B.3. Remarks on the upper integration limit}

To estimate the influence of the upper integration limit on the resulting contributions, the integral identity for $c_{f}(3.7)$ is evaluated for the $i Z P G_{M=0.5}^{+10 \mathrm{~K}}$ case with $y_{d}=0.2 \delta_{99}, 1.0 \delta_{99}$, $1.3 \delta_{99}$ and $2.0 \delta_{99}$. Results are depicted in figure 12 . As can be seen by comparison of figure $12(b, c)$, the choice of $y_{d}=1.0 \delta_{99}$ or of approximately $1.3 \delta_{99}$ does not influence the qualitative trends.

\section{B.4. Integral analysis}

For $c_{f}$, the constituent components of the spatial evolution term $c_{f}^{D}=\sum_{i=1}^{2} c_{f}^{D, i}$ are depicted in figure 13. For $c_{h}$, the constituent terms $c_{h}^{L}=\sum_{i=1}^{2} c_{h}^{L, i}, c_{h}^{T}=\sum_{i=1}^{6} c_{h}^{T, i}$ and $c_{h}^{D}=\sum_{i=1}^{3} c_{h}^{D, i}$ are depicted in figure 14. The index $i$ denotes the constituent components of the terms in (3.7) and (3.8) in ascending order from left to right. The sums of the 
Momentum and energy transfer in compressible TBLs

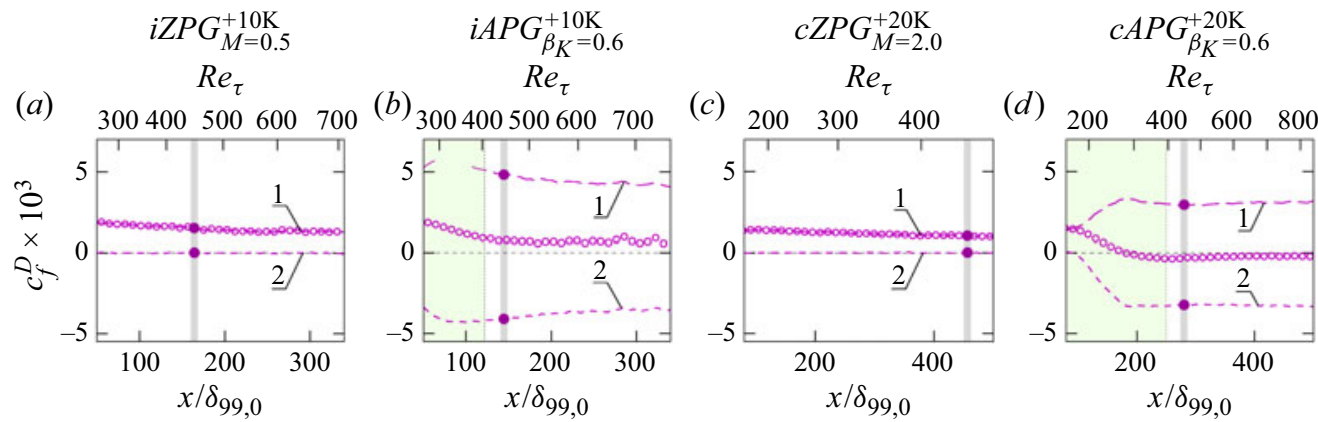

Figure 13. Composition of $c_{f}^{D}$ (open symbols) as given in figure $1(a-d)$ in its contributing parts $c_{f}^{D, 1}$ and $c_{f}^{D, 2}$ (lines); see (3.7). Values at $R e_{\tau}=450$ are marked for a visual comparison. Green regions in panels ( $\left.b, d\right)$ represent regions where the APG cases are not yet self-similar.
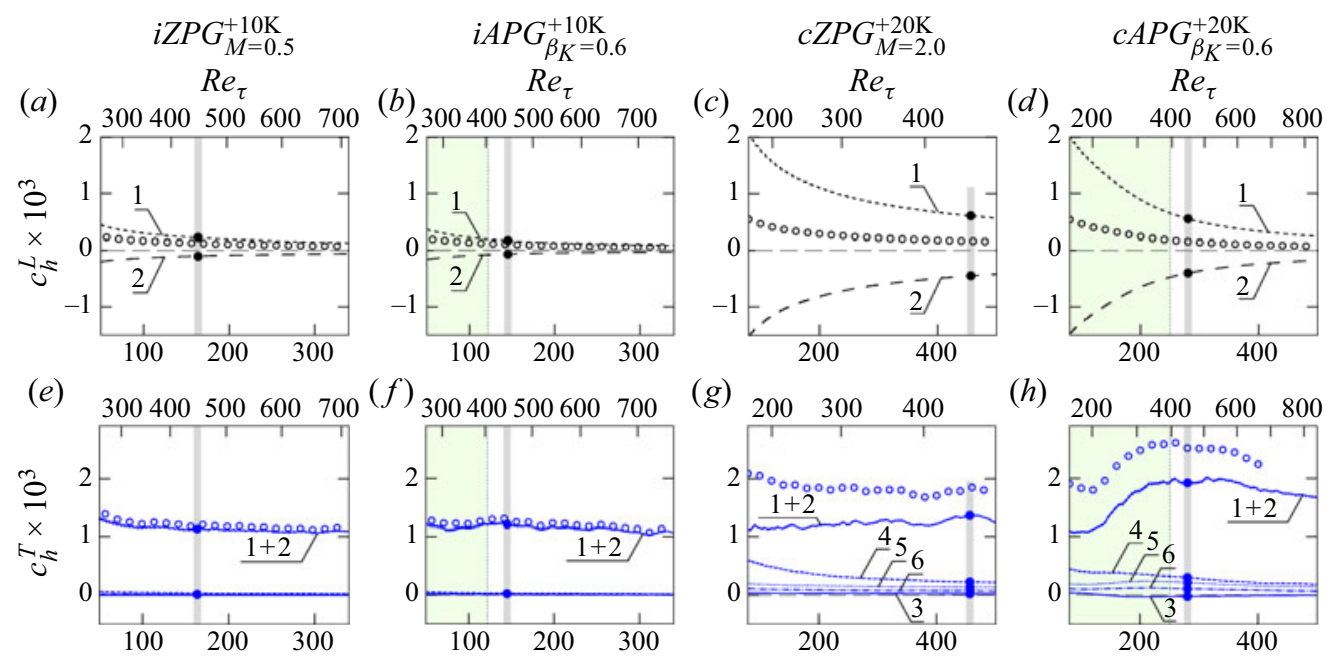

(h) $200 \quad 400 \quad 600 \quad 800$
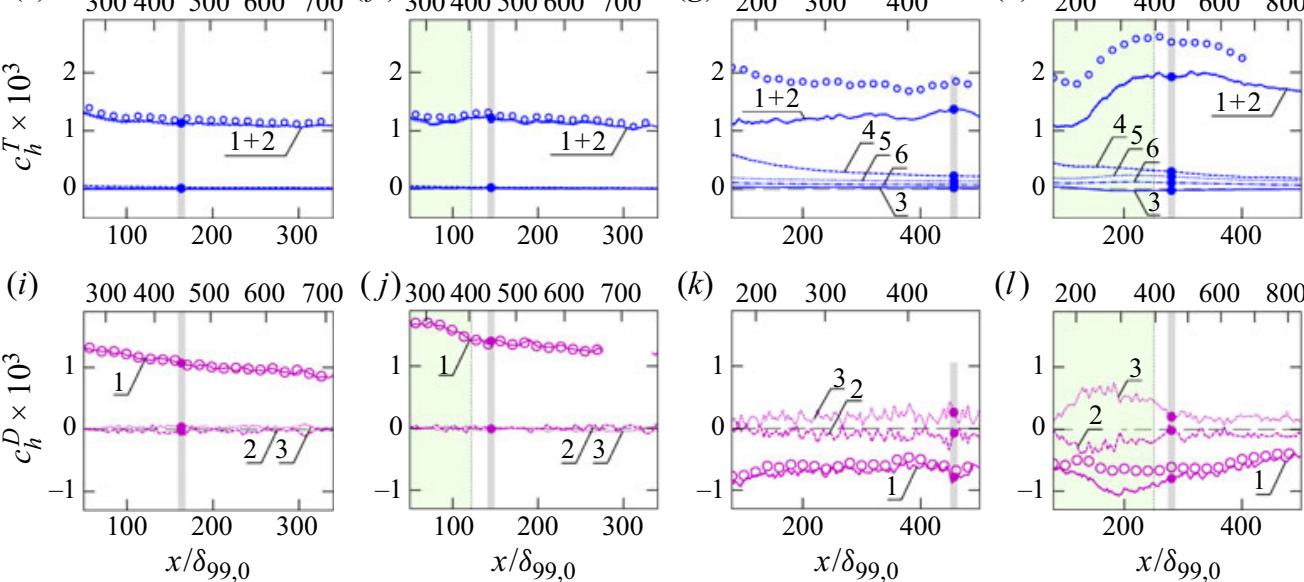

Figure 14. Composition of $c_{h}^{L}$ in panels $(a-d), c_{h}^{T}$ in panels $(e-h)$ and $c_{h}^{D}$ in panels (i-l) (open symbols) as given in figure $1(e-h)$ in its contributing parts $c_{h}^{L, i}, c_{h}^{T, i}$ and $c_{h}^{D, i}$; see (3.7). Values at $R e_{\tau}=450$ are marked for a visual comparison. Green regions represent regions where the APG cases are not yet self-similar.

individual terms are given as open circles in the respective plots and are identical to those given in figure 1; the constituent components of all terms are depicted as lines of different styles. 


\section{B.4.1. Evaluation of $c_{f}$}

For the $c_{f}$ identity (3.7), the spatial-development term $c_{f}^{D}=c_{f}^{D, 1}+c_{f}^{D, 2}$ consists of two contributing terms; $c_{f}^{D, 1}$ accounts for the mean convection in the streamwise direction, $c_{f}^{D, 2}$ accounts for the streamwise pressure gradient; see figure $13(a-d)$. Whereas $c_{f}^{D, 2}$ is (almost) equal to zero for the ZPG cases in panels $(a, c)$, its contribution approximately balances $c_{f}^{D, 1}$ for the pressure-gradient cases in panels $(b, d)$.

\section{B.4.2. Evaluation of $c_{h}$}

For both the sub- and supersonic cases in figure 14, the $c_{h}^{L}$ distributions in panels $(a-d)$ consist of a positive $c_{h}^{L, 1}$ term and a negative $c_{h}^{L, 2}$ term. Due to its dependency on $\bar{\mu} \partial \bar{T} / \partial y$ and $E c$, both terms strongly depend on the local Mach number. The $c_{h}^{T}$ distributions depicted in panels $(e-h)$ are dominated by $c_{h}^{T, 1}$ and $c_{h}^{T, 2}$; both terms are summed up. Due to the small absolute $E c$ number caused by the small Mach number and the near-adiabatic conditions, turbulent terms associated with the mean kinetic-energy transfer and turbulent kinetic-energy transfer, $c_{h}^{T, 3}-c_{h}^{T, 6}$, respectively, are almost negligible for the subsonic cases in panels $(e, f)$; compare figure 5 . For the supersonic cases in panels $(g, h)$, the contribution of $c_{h}^{T, 3}-c_{h}^{T, 6}$ makes up a substantial part of the overall contribution. The $c_{h}^{D}$ distributions depicted in panels $(i-l)$ are mainly influenced by the $c_{h}^{D, 1}$ term for the present cases.

\section{REFERENCES}

Arntz, A., Atinault, O. \& Merlen, A. 2015 Exergy-based formulation for aircraft aeropropulsive performance assessment: theoretical development. AIAA J. 53 (6), 1627-1639.

Bannier, A., Garnier, É. \& Sagaut, P. 2015 Riblet flow model based on an extended FIK identity. Flow Turbul. Combust. 95 (2-3), 351-376.

Bernardini, M. \& Pirozzoli, S. 2011 Inner/outer layer interactions in turbulent boundary layers: a refined measure for the large-scale amplitude modulation mechanism. Phys. Fluids 23 (6), 061701.

Deck, S., Renard, N., Laraufie, R. \& Weiss, P.-É. 2014 Large-scale contribution to mean wall shear stress in high-Reynolds-number flat-plate boundary layers up to $R e_{\theta}=13650$. J. Fluid Mech. 743, 202-248.

Drela, M. 2009 Power balance in aerodynamic flows. AIAA J. 47 (7), 1761-1771.

DuAn, L., BEEKMAN, I. \& MARTIN, M.P. 2011 Direct numerical simulation of hypersonic turbulent boundary layers. Part 3. Effect of Mach number. J. Fluid Mech. 672, 245-267.

DUAN, L. \& MARTIN, M.P. 2011 Direct numerical simulation of hypersonic turbulent boundary layers. Part 4. Effect of high enthalpy. J. Fluid Mech. 684, 25-59.

FAN, Y., CHENG, C. \& LI, W. 2019 Effects of the Reynolds number on the mean skin friction decomposition in turbulent channel flows. Appl. Maths Mech. 40 (3), 331-342.

FAN, Y., et al. 2020 Decomposition of the mean friction drag in adverse-pressure-gradient turbulent boundary layers. Phys. Rev. Fluids 5 (11), 114608.

Fernholz, H.H. \& Finley, P.J. 1980 A critical commentary on mean flow data for two-dimensional compressible turbulent boundary layers. Tech. Rep. DTIC Document.

Fukagata, K., Iwamoto, K. \& Kasagi, N. 2002 Contribution of Reynolds stress distribution to the skin friction in wall-bounded flows. Phys. Fluids 14 (11), L73-L76.

Gaviglio, J. 1987 Reynolds analogies and experimental study of heat transfer in the supersonic boundary layer. Intl J. Heat Mass Transfer 30 (5), 911-926.

Gibis, T., WenZel, C., Kloker, M. \& Rist, U. 2019 Self-similar compressible turbulent boundary layers with pressure gradients. Part 2. Self-similarity analysis of the outer layer. J. Fluid Mech. 880, 284-325.

Gomez, T., Flutet, V. \& Sagaut, P. 2009 Contribution of Reynolds stress distribution to the skin friction in compressible turbulent channel flows. Phys. Rev. E 79 (3), 035301.

GuArini, S.E., Moser, R.D., SharifF, K. \& Wray, A. 2000 Direct numerical simulation of a supersonic turbulent boundary layer at Mach 2.5. J. Fluid Mech. 414, 1-33. 


\section{Momentum and energy transfer in compressible TBLs}

Huang, P.G., Coleman, G.N. \& BRadshaw, P. 1995 Compressible turbulent channel flows: DNS results and modelling. J. Fluid Mech. 305, 185-218.

Iwamoto, K., Fukagata, K., Kasagi, N. \& Suzuki, Y. 2005 Friction drag reduction achievable by near-wall turbulence manipulation at high Reynolds numbers. Phys. Fluids 17 (1), 011702.

Kametani, Y. \& FuKagATA, K. 2011 Direct numerical simulation of spatially developing turbulent boundary layers with uniform blowing or suction. J. Fluid Mech. 681, 154-172.

Kametani, Y., Fukagata, K., Örlü, R. \& Schlatter, P. 2015 Effect of uniform blowing/suction in a turbulent boundary layer at moderate Reynolds number. Intl J. Heat Fluid Flow 55, 132-142.

VON KÁRMÁn, T. 1921 Über laminare und turbulente Reibung. Z. Angew. Math. Mech. 1, 233-252.

Li, W., Fan, Y., Modesti, D. \& Cheng, C. 2019 Decomposition of the mean skin-friction drag in compressible turbulent channel flows. J. Fluid Mech. 875, 101-123.

Mehdi, F., Johansson, T.G., White, C.M. \& Naughton, J.W. 2014 On determining wall shear stress in spatially developing two-dimensional wall-bounded flows. Exp. Fluids 55 (1), 1656.

Mendi, F. \& White, C.M. 2011 Integral form of the skin friction coefficient suitable for experimental data. Exp. Fluids 50 (1), 43-51.

Mellor, G.L. \& Gibson, D.M. 1966 Equilibrium turbulent boundary layers. J. Fluid Mech. 24 (2), 225-253.

Modesti, D., Pirozzoli, S., Orlandi, P. \& Grasso, F. 2018 On the role of secondary motions in turbulent square duct flow. J. Fluid Mech. 847, R1.

Morkovin, M.V. 1961 Effects of compressibility on turbulent flows. In Mécanique de la Turbulence (ed. A. Favre), pp. 367-380. CNRS.

PeEt, Y. \& SAgAut, P. 2009 Theoretical prediction of turbulent skin friction on geometrically complex surfaces. Phys. Fluids 21 (10), 105105.

RENARD, N. \& DECK, S. 2016 A theoretical decomposition of mean skin friction generation into physical phenomena across the boundary layer. J. Fluid Mech. 790, 339-367.

Smits, A.J. \& Dussauge, J.-P. 2006 Turbulent Shear Layers in Supersonic Flow. Springer Science \& Business Media.

So, R.M.C. 1994 Pressure gradient effects on Reynolds analogy for constant property equilibrium turbulent boundary layers. Intl J. Heat Mass Transfer 37 (1), 27-41.

Spalart, P.R. \& Strelets, M. 2000 Mechanisms of transition and heat transfer in a separation bubble. J. Fluid Mech. 403, 329-349.

Stroh, A., Hasegawa, Y., Schlatter, P. \& Frohnapfel, B. 2016 Global effect of local skin friction drag reduction in spatially developing turbulent boundary layer. J. Fluid Mech. 805, 303-321.

TANARRo, Á., VinUESA, R. \& SCHLATTER, P. 2020 Effect of adverse pressure gradients on turbulent wing boundary layers. J. Fluid Mech. 883, A8.

WALZ, A. 1959 Compressible turbulent boundary layers with heat transfer and pressure gradient in flow direction. J. Res. Natl Bur. Stand. 63, 53-70.

Wenzel, C., Gibis, T., KLOKER, M. \& Rist, U. 2019 Self-similar compressible turbulent boundary layers with pressure gradients. Part 1. Direct numerical simulation and assessment of Morkovin's hypothesis. J. Fluid Mech. 880, 239-283.

Wenzel, C., Gibis, T., Kloker, M. \& Rist, U. 2021 Reynolds analogy factor in self-similar compressible turbulent boundary layers with pressure gradients. J. Fluid Mech. 907, R4.

Wenzel, C., Selent, B., Kloker, M.J. \& Rist, U. 2018 DNS of compressible turbulent boundary layers and assessment of data/scaling-law quality. J. Fluid Mech. 842, 428-468.

Yoon, M., Ahn, J., Hwang, J. \& Sung, H.J. 2016 Contribution of velocity-vorticity correlations to the frictional drag in wall-bounded turbulent flows. Phys. Fluids 28 (8), 081702.

Zhang, C., DuAn, L. \& Choudhari, M.M. 2018 Direct numerical simulation database for supersonic and hypersonic turbulent boundary layers. AIAA J. 56 (11), 4297-4311.

Zhang, Y.-S., Bi, W.-T., Hussain, F. \& SHE, Z.-S. 2014 A generalized Reynolds analogy for compressible wall-bounded turbulent flows. J. Fluid Mech. 739, 392-420. 